\title{
SÉPARATION DES OSES, DES DI- ET TRIHOLOSIDES PAR CHROMATOGRAPHIE D'ÉCHANGE D'IONS
}

\author{
J. M. BESLE \\ avec la collaboration technique de Bernadette Lassalas \\ Station de Recherches sur l'Élevage des Ruminants, \\ Centre de Recherches de Clermont-Ferrand, I. N.R. A., \\ Theix, 63110 Beaumont
}

\section{RÉSUMÉ}

Nous avons étudié une méthode automatique de séparation et de dosage des mono- di- et triholosides d'origine biologique par chromatographie de leurs complexes avec les borates, sur résine échangeuse d'anions. Les glucides sont élués par un gradient de concentration en borate, chlorure de sodium et soude.

L'étude de l'influence du chargement de la résine, du gradient d'élution, de la température, du débit et du $\mathrm{pH}$ a conduit à préciser les conditions opératoires permettant une amélioration qualitative de la séparation et une plus grande rapidité d'élution pour une même résine (Technicon Chromobead S).

Les glucides sont dosés à la sortie de la colonne par la méthode à l'orcinol sulfurique. Un montage optimal de dosage à l'auto-analyseur est réalisé. L'influence de différents composés sur la réaction colorimétrique est mesurée.

L'évaluation des taux de récupération des glucides et de la reproductibilité de la méthode à diverses concentrations a permis de conclure que si les sucres sont partiellement dégradés dans la résine, ils le sont à un taux constant et ne s'épimérisent que de façon négligeable.

Lorsque la concentration des glucides est suffisamment élevée, la précision des dosages est satisfaisante puisque l'intervalle de confiance sur la moyenne de la constante de proportionnalité surface-concentration n'est pas supérieur à 3,o p. Ioo. Enfin, la reproductibilité des volumes de rétention est très bonne.

\section{INTRODUC'TION}

Pour étudier la composition en glucides des aliments et leur dégradation dans le tube digestif des animaux, il est nécessaire d'utiliser une méthode d'analyse rapide et précise des mélanges de mono- di- et triholosides. L'emploi des résines échangeuses d'ions a permis le développement de nombreuses méthodes de séparation des glucides. 
Ceux-ci peuvent être séparés directement par deux procédés. Le premier, par chromatographie d'adsorption, consiste à éluer les sucres avec de l'eau sur résine anionique (Hough, Priddie et Theobald, I960) ou cationique (Saunders, I968). Le deuxième, par chromatographie de partage, permet de les éluer, soit sur résine anionique avec un mélange propanol-eau (Adachi et Sugawara, I963) ou éthanol-eau (SAMUELson et SwENSON, I962), soit sur résine cationique avec un mélange éthanol-eau (Jonsson et Samueison, I966 et I967). Mis à part le procédé de Samuelson et StromBERG (I968) qui permet de séparer un mélange de mono- di- et triholosides en 5 heures, ces méthodes ne conviennent pas ou sont trop longues dans le cas d'échantillons comprenant ces trois catégories de sucres.

TABLEAU I

Principales variantes apportées à la méthode de KHYM et ZILL (I95I et 1952)

\begin{tabular}{|c|c|c|}
\hline But de la variante & Modification apportée & Auteurs principaux \\
\hline \multirow[t]{3}{*}{$\begin{array}{l}\text { Diminution du volume } \\
\text { nécessaire à l'élution }\end{array}$} & $\left\{\begin{array}{lll}\text { Augmentation } & \text { de } & \text { la } \\
\text { concentration } & \text { en } & \text { bo- } \\
\text { rate et du pH } & & \end{array}\right.$ & HaLLEN $(1960)$ \\
\hline & $\begin{array}{l}\text { Variation de la con- } \\
\text { centration en borate, } \\
\text { du pH, de la tempéra- } \\
\text { ture, du débit d'élution } \\
\text { des caractéristiques de } \\
\text { la colonne et de la ré- } \\
\text { sine. }\end{array}$ & 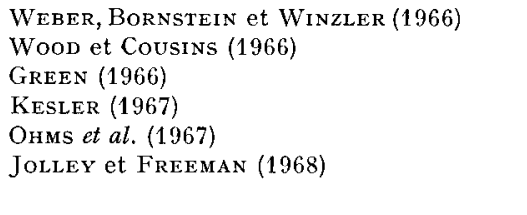 \\
\hline & $\begin{array}{c}\text { Addition de chlorure } \\
\text { de sodium }\end{array}$ & $\begin{array}{l}\text { Syamananda, Staples et Block (1962) } \\
\text { Catravas (1966) } \\
\text { Mundie, Cheshire et Bacon (1968) } \\
\text { LeE, McKelvy et LANG (1969) } \\
\text { Floridi (1971) }\end{array}$ \\
\hline $\begin{array}{l}\text { Amélioration de la symé- } \\
\text { trie des pics }\end{array}$ & $\begin{array}{c}\text { Suppression de chlorure } \\
\text { de sodium }\end{array}$ & KesLer (1967) \\
\hline \multirow[t]{2}{*}{$\begin{array}{l}\text { Élimination des réac- } \\
\text { tions secondaires des } \\
\text { glucides dans la résine }\end{array}$} & $\begin{array}{l}\text { pH neutre, addition de } \\
\text { glycérol }\end{array}$ & $\begin{array}{l}\text { Walborg, ChristensSon et Gardell (1965) } \\
\text { WaLborg et Lanz (1968) } \\
\text { Touze et AURIoL (1970) }\end{array}$ \\
\hline & $\begin{array}{l}\text { pH neutre, addition de } \\
\text { butanediol }\end{array}$ & $\begin{array}{l}\text { WALBorg, Ray et OHRBERG (1969) } \\
\text { WALBORG et Kondo }(1970)\end{array}$ \\
\hline
\end{tabular}

Les glucides ne sont pas normalement ionisés; néanmoins, ils forment des anions en se complexant avec les borates (BOESEKEN I949), les tungstates ou les molybdates (ANGus et al., I964). La réaction entre les borates et les glucides a permis de

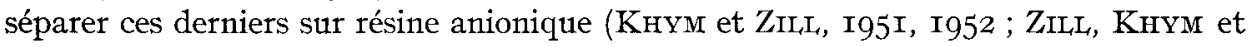
Cheniae, I953). Ce procédé a ensuite été repris une dizaine d'années plus tard et constamment amélioré. Le tableau I montre les modifications qui ont été apportées à la méthode originale. Le procédé utilisant un $\mathrm{pH}$ neutre et l'addition d'alcool limite 
les risques de dégradation et de transformation des glucides dans la colonne mais il conduit à des séparations relativement longues ( 7 à 20 heures) et nécessite l'emploi, pour le dosage, d'une réaction colorimétrique insensible à l'alcool. La réaction à l'aniline-acide acétique-acide phosphorique répond à cette nécessité mais elle doit être réalisée en continu à $\mathrm{I} 20^{\circ} \mathrm{C}$ sous pression (WALBORG et KONDO, I970). La méthode à la cystéine sulfurique utilisée par TOUzE et AURIOL (I970) convient également mais elle est beaucoup moins sensible que les réactions à l'orcinol, à l'anthrone et au phénol.

Récemment, LEE, McKeivy et LANG (I969) puis FLoridi (I97I) ont réalisé une élution complète, respectivement en 5 heures et 4 heures Io minutes ; mais les premiers n'étudient pas les diholosides et séparent mal le mannose du ribose, le deuxième n'élue que deux diholosides avec le procédé le plus rapide (saccharose et maltose).

En nous inspirant de la méthode de Catravas (I966), nous avons mis au point un procédé permettant une meilleure séparation qualitative des glucides, en un temps beaucoup plus court. Nous avons déterminé les caractéristiques opératoires en fonction du type de mélange de glucides à analyser. Enfin, nous avons étudié la réaction colorimétrique du dosage des sucres élués et précisé les limites de la méthode.

\section{MÉTHODE PROPOSÉE}

$$
\text { I. - Principe }
$$

Les glucides sont séparés sur une colonne de résine échangeuse d'anions, thermostatée à $55^{\circ} \mathrm{C}$, par un gradient de concentration croissante en borate, en chlorure de sodium et en soude. Ils sont ensuite dosés en continu dans l'effluent par la réaction à l'orcinol sulfurique.

\section{2. - Matériel et véactifs]}

a) Appareillage.

L'ensemble de l'appareillage est décrit dans la figure I. Deux modifications ont été apportées au schéma habituellement utilisé :

- Un combinateur Crouzet $\left.{ }^{(}\right)$et des minivannes montées sur cet appareil permettent d'automatiser le cycle de la colonne : régénération-équilibration-élution.

- Les gaz dissous dans l'éluant sont éliminés par le système représenté sur le schéma : la pompe à circulation du bain marie envoie de l'huile très fluide (Ioo csk) à $95^{\circ} \mathrm{C}$ autour d'une bobine dans laquelle passe l'éluant. Les gaz dissous s'échappent et s'accumulent dans un petit tube incliné. A la fin de chaque chromatographie, ce dernier est purgé des gaz qui s'y sont accumulés. De cette manière, ils sont éliminés en continu.

b) Colonnes de résines utilisées.

- Pour la chromatographie.

Utiliser la résine échangeuse d'anions forte Technicon du Type Chromobead $\mathrm{S}$ dont les grains ont une taille moyenne de 20 microns. La hauteur de la colonne de résine est d'environ $68 \mathrm{~cm}$,

(1) Crouzet, Route d'Alixan, B. P. I38, 26 - Valence. 
son diamètre de $6 \mathrm{~mm}$. Les impuretés grossières de l'éluant sont éliminées avant la micropompe par un filtre en laine de verre, les plus fines sont arrêtées avant la colonne par un disque fritté en téflon. La perte de charge occasionnée par ce dispositif est négligeable.

\section{- Pour la purification.}

Utiliser les résines échangeuses d'anions $\mathrm{AG} 2 \times 8$, 50-100 mesh, Biorad $\left({ }^{2}\right)$ et échangeuses de cations AG $50 \mathrm{~W} \times 8,200-400$ mesh, Biorad $\left({ }^{2}\right)$. Les colonnes ont un diamètre interne de $16 \mathrm{~mm}$ et une hauteur utile de $20 \mathrm{~cm}$, un piston $\left(^{3}\right)$ arrive jusqu'au niveau de la résine et élimine le volume mort.

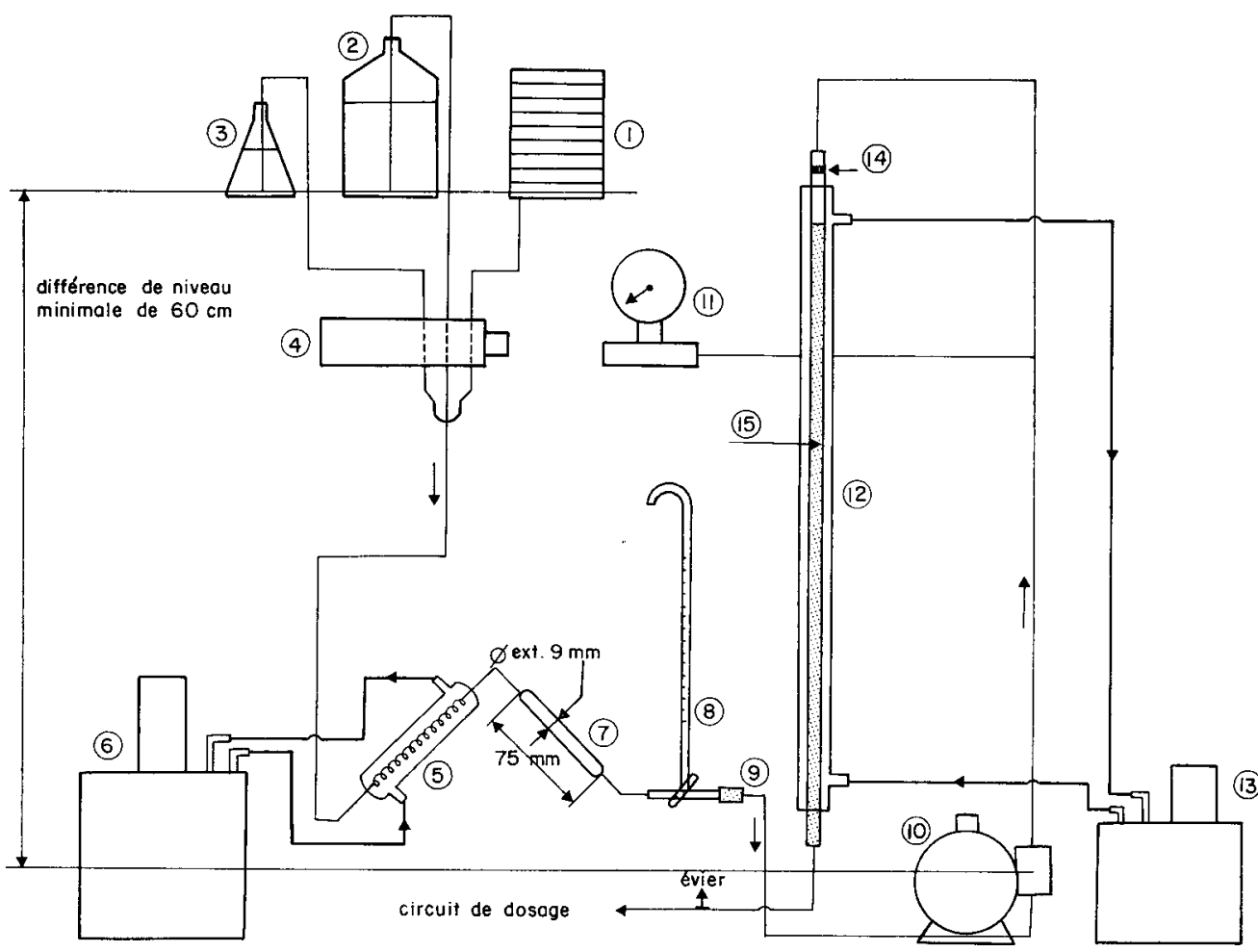

FIG. I. - Appareillage servant à la chromatographie des glucides

(I) Autograd à 9 chambres Technicon

(2) Solution de régénération

(3) Solution d'équilibration

(4) Moteur Crouzet à mini contacts et minivannes

(5) Bobine thermostatée à $95^{\circ} \mathrm{C}$

(6) Bain-Marie du circuit de dosage avec thermostat à circulation Braun Thermomix II, huile SISS, SI 200, I00 c s k

(7) Chambre d'accumulation des gaz

(8) Débitmètre Technicon

(9) Filtre en laine de verre

(Io) Micropompe haute pression Milton Roy

(II) Manomètre de pression Technicon

(I2) Colonne thermostatée $750 \times 6 \mathrm{~mm}$ Technicon

(x3) Thermostat à circulation Haake $\mathrm{F} \mathrm{E}$

(I4) Filtre fin d'entrée de l'éluant en téflon fritté

(I5) Résine (environ $68 \mathrm{~cm}$ de hauteur) Chromobead S Technicon

(2) Touzart et Matignon, 3, rue Amyot, Paris, $5^{\mathrm{e}}$.

(3) Farmacia, 5-75125, Uppsala, I, Suède. 
c) Produits chimiques et réactifs.

- Pour la purification des échantillons.

- Solution de Baryte RP (4) saturée $(0,3 \mathrm{~N})$.

- Sulfate de Zinc RP ( $\left.{ }^{4}\right)$ en solution ro p. roo.

- Acide acétique RP $\left({ }^{4}\right)$.

- Soude RP ( $\left.{ }^{4}\right)$ en solution 3 N.

- Acide chlorhydrique RP $\left(^{4}\right)$ en solution $3 \mathrm{~N}$.

\section{- Pour le dosage.}

- Orcinol ou 3,5 dihydroxy toluène RP ( $\left.{ }^{4}\right)$ en solution I p. Ioo dans de l'eau bidistillée et filtrée.

- Acide sulfurique p.a. (5) $\mathrm{d}=\mathrm{I}, 84$ en solution $7^{\circ} \mathrm{p}$. Ioo v/v dans de l'eau bidistillée.

\section{- Pour la chromatographie.}

Toutes les solutions envoyées sur la colonne sont préparées avec de l'eau bidistillée puis filtrées sur filtre millipore $0,2 \mu$.

- Tampons d'élution : les préparer à l'aide d'acide borique p.a. $\left({ }^{5}\right)$ et de soude RP $\left(^{4}\right)$ en solution $2 \mathrm{~N}$ et I $2 \mathrm{~N}$. Leur composition est indiquée au tableau 2. Le tampon I sert à l'équilibration de la résine.

- Tétraborate de potassium $\left({ }^{6}\right)$ en solution Io p. 100.

- Soude RP (4) N pour le lavage de la résine.

- Acide chlorhydrique RP (4) N pour le lavage de la résine.

- Brij $35\left(^{\mathbf{1}}\right)$ : préparer une solution concentrée à $30 \mathrm{p}$. 1oo, en diluer o,5 $\mathrm{ml}$ dans I 1 d'acide chlorhydrique $\mathrm{N}$.

Le tampon $\mathrm{I}$, les solutions de soude et d'acide chlorhydrique sont conservés à $4^{\circ} \mathrm{C}$, tous les autres réactifs se conservent à température ambiante.

- Glucides étalons.

- Glucides sigma $\left({ }^{7}\right)$ : D $(+)$ : cellobiose ; D (-) : fructose ; D (+) : fucose ; D (+) : galactose ; 2 : désoxygalactose ; $\mathrm{D}(+)$ : glucose; $\mathrm{D}$ : méthylglucoside, lactose hydrate, maltose hydrate ; $\mathrm{D}(+)$ : mannose ; 2 : désoxy-D-ribose ; $\mathrm{L}(-)$ : sorbose ; $\mathrm{D}(+)$ : xylose.

- Glucides nbc $\left(^{8}\right)$ (Nutritional Biochemicals Corporation) : D $(-)$ : arabinose ; D $(+)$ : raffinose penta-hydrate; D $(-)$ : ribose ; $\mathrm{L}(+)$ : rhamnose hydrate ; $\mathrm{D}(+)$ : saccharose.

- Glucide touzart et matignon $\left({ }^{2}\right): D(+)$ : tréhalose hydrate.

Les solutions mères de glucides étalons sont préparées dans de l'eau bidistillée avec une goutte d'acide chlorhydrique concentré par Ioo $\mathrm{ml}$ et conservées à - $20^{\circ} \mathrm{C}$. Avant 1'emploi, on les dilue ro fois dans du tampon $I$.

Le standard externe utilisé pour étalonner le circuit de dosage est une solution de saccharose : $5 \mu \mathrm{g} / \mathrm{ml}$ dans de l'eau bidistillée et conservée à - $20^{\circ} \mathrm{C}$. La solution mère de roo $\mu \mathrm{g} / \mathrm{ml}$ est préparée dans de l'eau bidistillée avec une goutte d'HCl concentré pour Ioo $\mathrm{ml}$ et de chloroforme (MoNTREUIL et SPIK, I963). Elle peut être conservée plusieurs mois à - $20^{\circ} \mathrm{C}$.

\section{3. - Mode opératoive}

a) Préparation des échantillons (cas d'extraits aqueux).

Suivant leur type, les échantillons aqueux peuvent être utilisés tels quels ou ultrafiltrés (filtre millipore $0, \mathrm{I} \mu$ ). Dans certains cas, il est nécessaire, soit de les déféquer soit de les purifier sur résines, soit de leur faire subir successivement les deux traitements. De toute manière, les solutions glucidiques doivent être mises en milieu borate o, I M, pH 8, avant chromatographie de façon à avoir une séparation optimale. Nous décrirons le schéma de purification le plus complet qui permet d'éviter les effets de sel (JoLLEY et FREEMAN, I968) et les interférences dans la réaction colorimétrique. Il a été vérifié qu'aucune perte de glucides n'a lieu tout au long du processus de purification.

(4) Prolabo, I2, rue Pelée, Paris, I $\mathbf{e}$

(5) Riedel de Haen, 30:6, Seelze, Allemagne.

$\left({ }^{8}\right) \mathrm{BDH}$ Chemical Ltd, Overseas Division, Poole, Angleterre BH $124 \mathrm{NN}$

(7) Sigma 3500 Dekalb St. St-Louis Mo. 631 I8. U. S. A.

(8) Nutritional Biochemicals Corporation, 2620 I Miles Road, Cleveland, Ohio 44r28, U. S. A. 
Mettre les résines échangeuses d'anions et de cations respectivement sous forme acétate et $\mathrm{H}^{(+)}$selon la méthode de MontreurL, SPIK et Konarska (I963) dans laquelle nous remplaçons l'acide formique par l'acide acétique. Elles peuvent être mises đans 2 colonnes en série avec 2 pistons arrivant au niveau des résines ou bien superposées dans une seule, la résine anionique formant la couche inférieure. Dans le deuxième cas, on n'utilise qu'une seule colonne mais il faut ensuite séparer les 2 résines pour les régénérer.

Ajouter à $7 \mathrm{ml}$ d'échantillon $7 \mathrm{ml}$ de sulfate de zinc à Io $\mathrm{p}$. Ioo et $\mathrm{I}_{4} \mathrm{ml}$ de baryte saturée, filtrer. Une quantité de $3 \mathrm{~g}$ de chaque résine humide est suffisante pour purifier plusieurs fois Io $\mathrm{ml}$ de défécat (Déterminer le volume total pouvant être purifié suivant la concentration en ions de chaque type d'échantillon).

Injecter ro $\mathrm{ml}$ de filtrat sur les résines à travers le piston au débit de $5 \mathrm{ml} / \mathrm{mn}$ à l'aide d'une seringue ou mieux, d'une pompe péristaltique. Éluer ensuite à l'eau bidistillée au débit de $5 \mathrm{ml} / \mathrm{mn}$ assuré par un vase de Mariotte ou une pompe péristaltique. Recueillir la première fraction dans une fiole de $50 \mathrm{ml}$, elle contient la totalité des glucides. Évaporer à sec sous vide ou lyophiliser puis reprendre dans du tampon $\mathbf{I}$ de façon à avoir une concentration convenable en glucides.

\section{b) Préparation de la colonne.}

Le gonflement de la résine étant très faible avec l'acide chlorhydrique (GotTliEB et GrEgor, I954; GREGOR, BELLE et MARCus, I954), charger la colonne avec la résine sous forme $\mathrm{Cl}^{(-)}$dans une solution à $55^{\circ} \mathrm{C}$ contenant de l'acide chlorhydrique $\mathrm{N}$ et du Brij 35 à o, 5 p. Iooo dont on a préalablement éliminé les gaz dissous sous vide. Le mode opératoire est ensuite le même que celui proposé par MoORE, SPACKMAN et STEIN (I958). La résine étant tassée, faire passer successivement à l'aide de la micropompe réglée sur le débit d'utilisation de la colonne, de l'eau pendant 2 heures puis la solution de tétraborate ro p. Ioo jusqu'à ce qu'il n'y ait plus de chlorure dans l'effluent de colonne, enfin du tampon I pour équilibrer la résine. De cette manière, on obtient une bonne résolution dès les premiers chromatogrammes et le volume de résine ne change pas, même après un nombre important de chromatographies.

La micropompe étant réglée à un débit de $0,7 \mathrm{ml} / \mathrm{mn}$, régénérer la résine pendant 4 heures durant la nuit avec la solution de tétraborate de potassium à Io p. Ioo. Ensuite, équilibrer pendant I h 30 avec du tampon I. Entre deux chromatographies successives de 2 heures, au débit de $\mathrm{I} \mathrm{ml} / \mathrm{mn}$, régénérer pendant $\mathrm{I} h 30$ à la fin de la première séparation puis équilibrer pendant $30 \mathrm{mn}$. Changer périodiquement la partie supérieure de la résine sur une épaisseur de $\mathbf{~} \mathrm{cm}$ car elle brunit progressivement (OHMs et al., I967). L'ultrafiltration des solutions et le disque en téflon fritté à l'entrée de la colonne diminuent considérablement le brunissement mais il subsiste encore.

Enfin, lorsque le pouvoir de résolution n'est plus satisfaisant ou que la perte de charge augmente trop, purifier la résine en colonne par le passage successif de $250 \mathrm{ml}$ de chacune des solutions suivantes : eau, acide chlorhydrique $\mathrm{N}+$ Brij, eau, soude $\mathrm{N}$, eau, tétraborate ro p. Ioo, tampon I + Brij, puis tampon I jusqu'à ce que tout le Brij soit éliminé. Pendant le passage de la soude et tant que la résine est sous forme $\mathrm{OH}^{(-)}$, donc très sensible à la température, il convient de maintenir la température de la colonne à $40^{\circ} \mathrm{C}$. La résine non utilisée est conservée à $4^{\circ} \mathrm{C}$.

\section{c) Introduction de l'échantillon.}

Chauffer l'échantillon à $55^{\circ} \mathrm{C}$ pendant cinq minutes avant l'injection. Avec une seringue de $25^{\circ} \mu \mathrm{l}$, déposer par pesée une quantité exacte d'environ $\mathrm{O}, \mathrm{I} \mathrm{g}$, la faire pénétrer dans la résine sous pression d'air comprimé, rincer avec o, I $\mathrm{ml}$ de tampon I et éluer.

Auparavant, pomper dans le circuit analytique un standard externe de $5 \mu \mathrm{g} / \mathrm{ml}$ de saccharose pendant $3 \mathrm{mn}$.

\section{d) Élution de l'échantillon.}

Suivant le mélange glucidique à analyser, différents gradients de concentration doivent être utilisés. Leur composition et les conditions de chromatographie (débit et température) sont indiquées au tableau 2. Les résultats obtenus sont reproduits (fig. $2 a$ et $2 b$ ).

- Le gradient $\mathrm{B}$ sépare mieux le fructose de l'arabinose et convient particulièrement à un mélange de nombreux mono- di- et triholosides qu'il peut séparer en $5 \mathrm{~h} 30$.

- Le gradient $C$ permet de séparer I 3 sucres en 4 h et de faire deux chromatographies dans la journée en procédant à une régénération rapide à la fin de la première.

-. Le gradient $\mathrm{D}$ permet de faire une séparation en $2 \mathrm{~h}$; il convient dans le cas d'un mélange ne comportant qu'un plus petit nombre de glucides. 


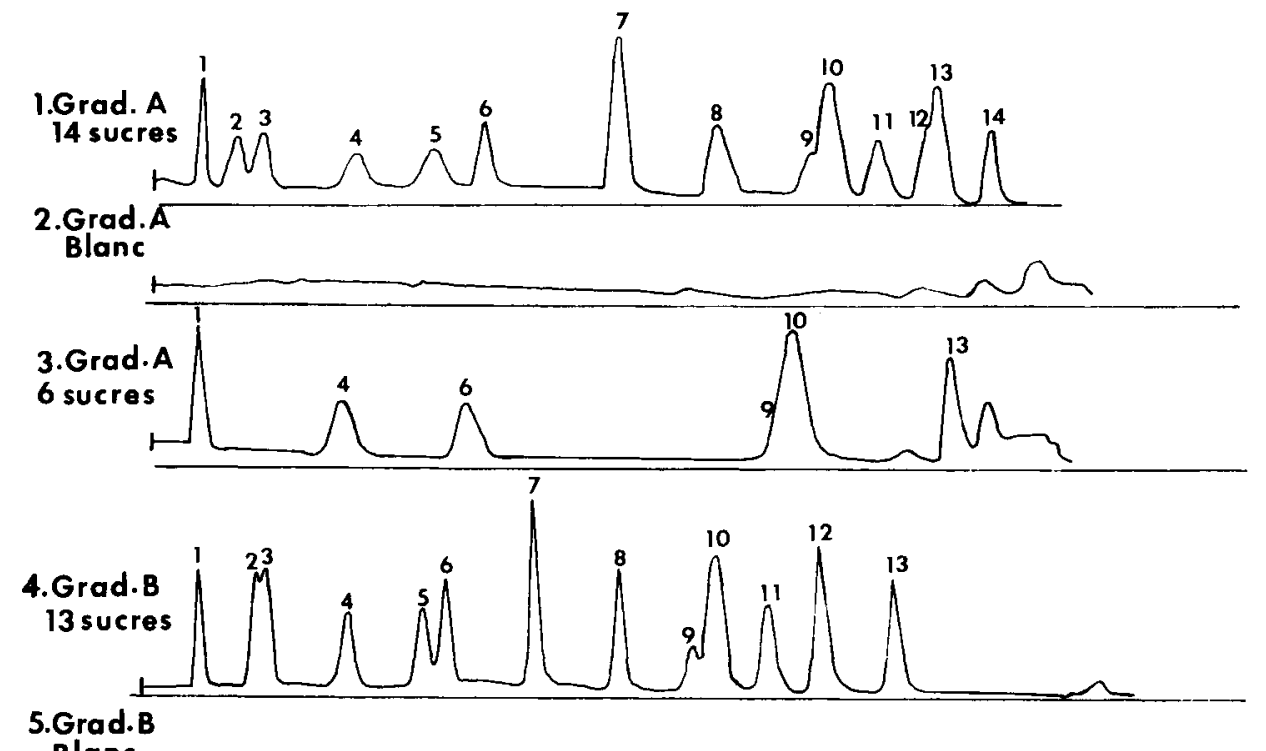

\section{Grad.B \\ Blane}

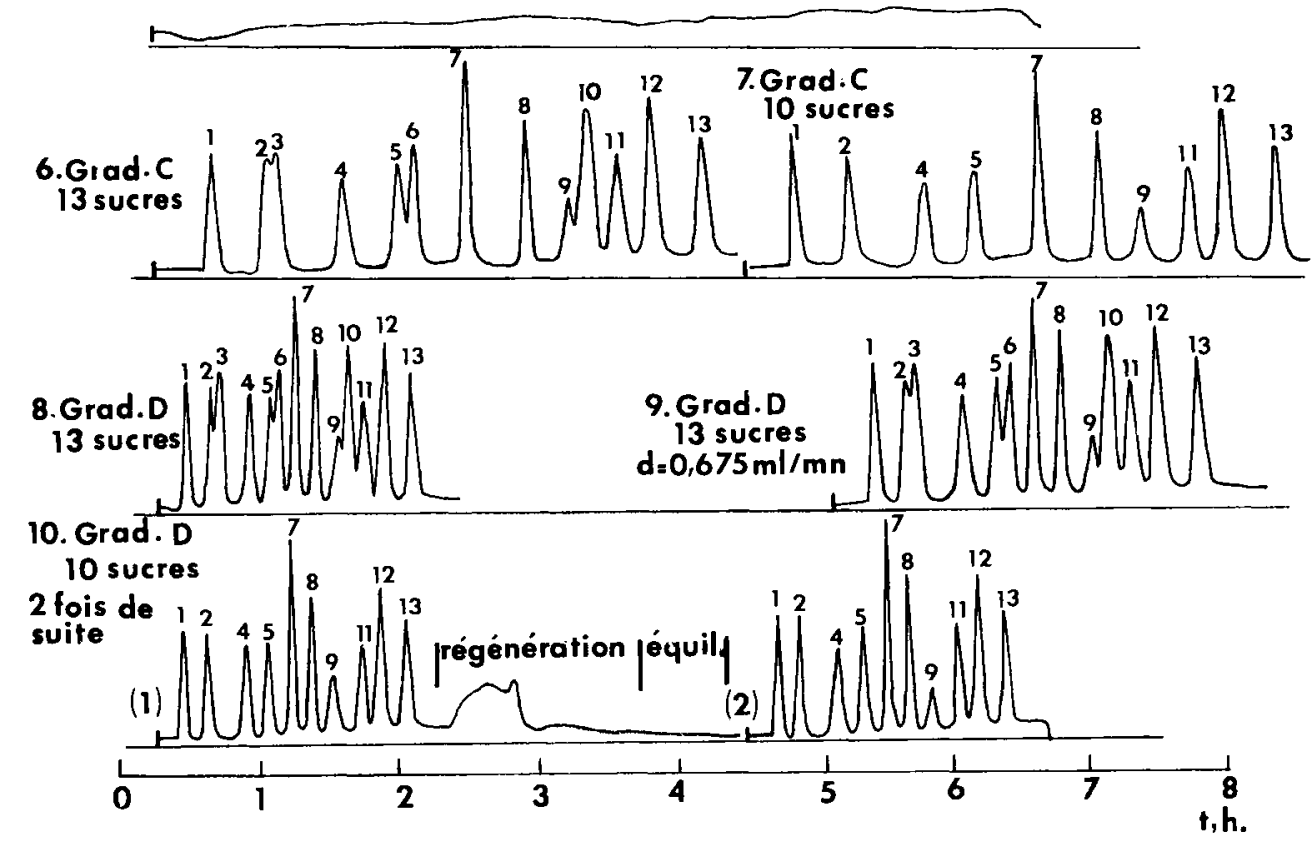

FIG. 2 a. - Influence du gradient d'elution (cf. composition des gradients tabl. 2)

Le décalage entre le zéro de l'élution (échelle du bas) et le départ du chromatogramme correspond au volume mort qui est déduit à chaque fois.

Toute les chromatographies ont été réalisées en déposant o, $\mathrm{r} \mathrm{ml}$ de solutions contenant $200 \mu \mathrm{g} / \mathrm{ml}$ de chaque glucide.

Chromatogramme 3 : deux artefacts proviennent du gradient. Il a été réalisé, ainsi que les autres chromatogrammes, un an après la séparation ( $\mathrm{I}$ ), avec la résine déjà usagée. L'arabinose et le fructose sont moin bien séparés qu'en (I).

Chromatogramme Io : deux séparations sont réalisées en $6 \mathrm{~h} 30$.

I : saccharose ; 2 : raffinose ; 3 : cellobiose; 4 maltose ; 5 : lactose ; 6 : rhamnose ; 7 : ribose ; 8 : mannose ; 9 : fructose ; Io : arabinose ; II : galactose ; III : sorbose ; I2 : xylose ; I3 : glucose. 
TABLEAU 2

Composition des gradients d'élution (Varigrad à 9 chambres)

Les volumes dans les chambres sont exprimés en $\mathrm{ml}$

\begin{tabular}{|c|c|c|c|c|c|c|c|c|c|c|}
\hline & \multirow{2}{*}{ Solutions ( ${ }^{1}$ ) } & \multicolumn{9}{|c|}{ Chambres } \\
\hline & & 1 & 2 & 3 & 4 & 5 & 6 & 7 & 8 & 9 \\
\hline $\begin{array}{c}\text { Gradient A } \\
\text { CATravas, } 1966 \\
\text { débit : } \\
0,75 \mathrm{ml} / \mathrm{mn} \\
t: 53,5^{\circ} \mathrm{C}\end{array}$ & $\begin{array}{l}\mathrm{H}_{3} \mathrm{BO}_{3} 0,1 \mathrm{M} \mathrm{pH} 8,0 \text { (tampon 1) } \ldots \\
\mathrm{H}_{3} \mathrm{BO}_{3} 0,1 \mathrm{M}+\mathrm{NaCl} 0,05 \mathrm{M} \mathrm{pH} 8,0 \\
\mathrm{H}_{3} \mathrm{BO}_{3} 0,1 \mathrm{M}+\mathrm{NaCl} 0,1 \mathrm{M} \mathrm{pH} 8,0 . \\
\mathrm{H}_{3} \mathrm{BO}_{3} 0,2 \mathrm{M} \mathrm{pH} 8,0 \ldots \ldots \ldots \ldots \ldots \\
\mathrm{H}_{3} \mathrm{BO}_{3} 0,2 \mathrm{M}+\mathrm{NaCl} 0,2 \mathrm{M} \mathrm{pH} 9,5\end{array}$ & 50 & 50 & $\begin{array}{l}25 \\
25\end{array}$ & $\begin{array}{l}20 \\
30\end{array}$ & $\begin{array}{l}25 \\
25\end{array}$ & $\begin{array}{l}25 \\
25\end{array}$ & $\begin{array}{l}25 \\
25\end{array}$ & 50 & 50 \\
\hline $\begin{array}{l}\text { Gradient B } \\
\text { débit : } \\
0,675 \mathrm{ml} / \mathrm{mn} \\
t: 55^{\circ} \mathrm{C}\end{array}$ & $\begin{array}{l}\mathrm{H}_{3} \mathrm{BO}_{3} 0,1 \mathrm{M} \text { pH } 8,0 \ldots \ldots \ldots \ldots \\
\mathrm{H}_{3} \mathrm{BO}_{3} 0,6 \mathrm{M} \text { pH } 9,5 \ldots \ldots \ldots \ldots \ldots \\
\mathrm{H}_{3} \mathrm{BO}_{3} 0,6 \mathrm{M}+\mathrm{NaCl} 0,1 \mathrm{M} \text { pH } 9,5 \\
\mathrm{H}_{3} \mathrm{BO}_{3} 0,6 \mathrm{M}+\mathrm{NaCl} 0,2 \mathrm{M} \text { pH } 9,5\end{array}$ & 45 & 45 & 45 & 45 & $\begin{array}{l}30 \\
15\end{array}$ & 45 & 45 & 45 & 45 \\
\hline $\begin{array}{l}\text { Gradient C } \\
\text { débit : } \\
0,675 \mathrm{ml} / \mathrm{mn} \\
t: 55^{\circ} \mathrm{C}\end{array}$ & $\begin{array}{l}\mathrm{H}_{3} \mathrm{BO}_{3} 0,1 \mathrm{M}+\mathrm{NaCl} 0,05 \mathrm{M} \mathrm{pH} 8,0 \\
\mathrm{H}_{3} \mathrm{BO}_{3} 0,1 \mathrm{M}+\mathrm{NaCl} 0,1 \mathrm{M} \mathrm{pH} 8,0 . \\
\mathrm{H}_{3} \mathrm{BO}_{3} 0,6 \mathrm{M}+\mathrm{NaCl} 0,05 \mathrm{M} \mathrm{pH} 9,5 \\
\mathrm{H}_{3} \mathrm{BO}_{3} 0,6 \mathrm{M}+\mathrm{NaCl} 0,1 \mathrm{M} \mathrm{pH} 9,5 . \\
\mathrm{H}_{3} \mathrm{BO}_{3} 0,6 \mathrm{M}+\mathrm{NaCl} 0,2 \mathrm{M} \mathrm{pH} 9,5 .\end{array}$ & 45 & 45 & 45 & 45 & $\begin{array}{l}30 \\
15\end{array}$ & 45 & 45 & 45 & 45 \\
\hline $\begin{array}{c}\text { Gradient } \mathrm{D} \\
\text { débit }: 1 \mathrm{ml} / \mathrm{mn} \\
t: 55^{\circ} \mathrm{C}\end{array}$ & $\begin{array}{l}\mathrm{H}_{3} \mathrm{BO}_{3} 0,1 \mathrm{M}+\mathrm{NaCl} 0,05 \mathrm{M} \mathrm{pH} 8,0 \\
\mathrm{H}_{3} \mathrm{BO}_{3} 0,1 \mathrm{M}+\mathrm{NaCl} 0,1 \mathrm{M} \mathrm{pH} 8,0 . \\
\mathrm{H}_{3} \mathrm{BO}_{3} 0,6 \mathrm{M}+\mathrm{NaCl} 0,05 \mathrm{M} \mathrm{pH} 9,5 \\
\mathrm{H}_{3} \mathrm{BO}_{3}+0,6 \mathrm{M}+\mathrm{NaCl} 0,1 \mathrm{M} \mathrm{pH} 9,5 \\
\mathrm{H}_{3} \mathrm{BO}_{3} 0,6 \mathrm{M}+\mathrm{NaCl} 0,2 \mathrm{M} \mathrm{pH} 9,5 .\end{array}$ & 25 & 25 & 25 & 25 & $\begin{array}{l}17 \\
8\end{array}$ & 25 & 25 & 25 & 25 \\
\hline
\end{tabular}

(1) Le pH est atteint avec de la soude 2 ou $12 \mathrm{~N}$ suivant qu'il est de 8,0 ou de 9,5 .

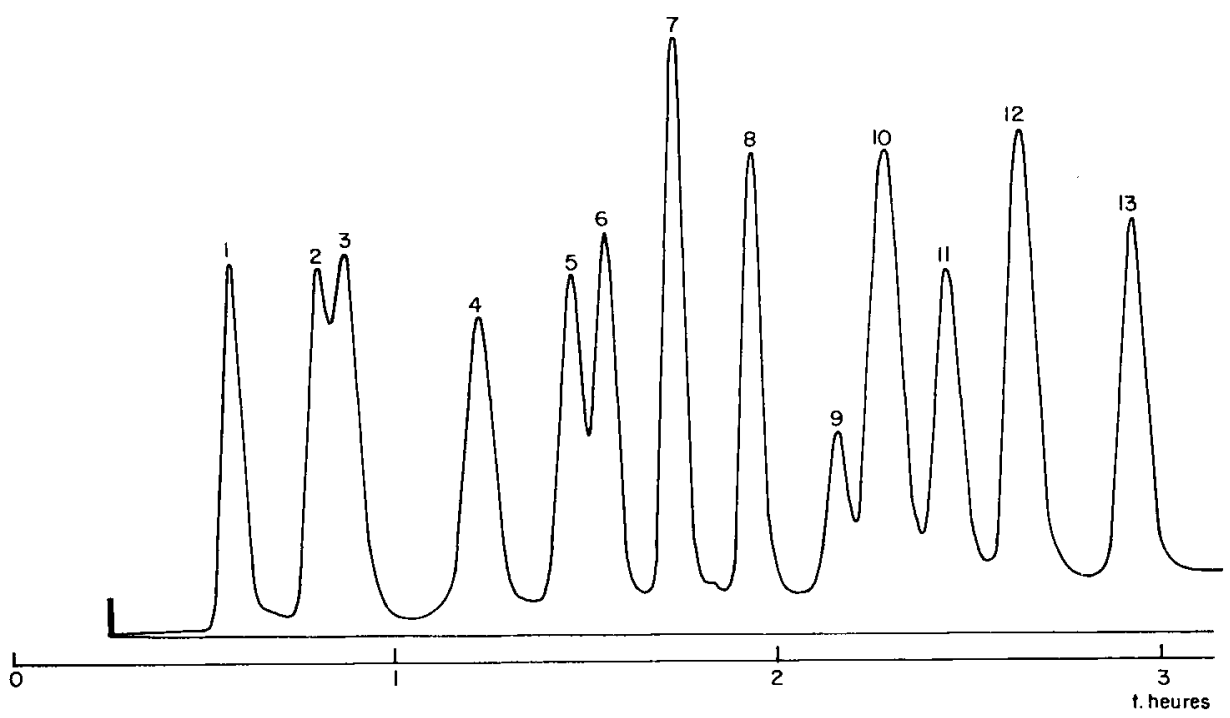

Fig. 2 b. - Séparation de 13 sucres en 3 heures

Élution par le gradient $\mathrm{D}$ au débit de $0,675 \mathrm{ml} / \mathrm{mn}$

Les conditions opératoires sont les mêmes que pour la fig. $2 a$ 


\section{e) Dosage des sucres.}

Le procédé à l'orcinol sulfurique a été choisi pour sa reproductibilité, sa sensibilité et parce que son facteur de réponse vis-à-vis des divers sucres est moins variable que celui des autres méthodes comparables (anthrone sulfurique, phénol sulfurique, cystéine sulfurique). Décrit par Tillmans et Phirippi (I929), il est basé sur la réaction de Bial (I902) et a été adapté à l'autoanalyseur par KEsLER ( ${ }^{967}$ ). Nous avons réalisé un montage analytique optimal (fig. 3) qui limite la consommation de réactifs tout en permettant une bonne sensibilité et la reproductibilité des dosages.

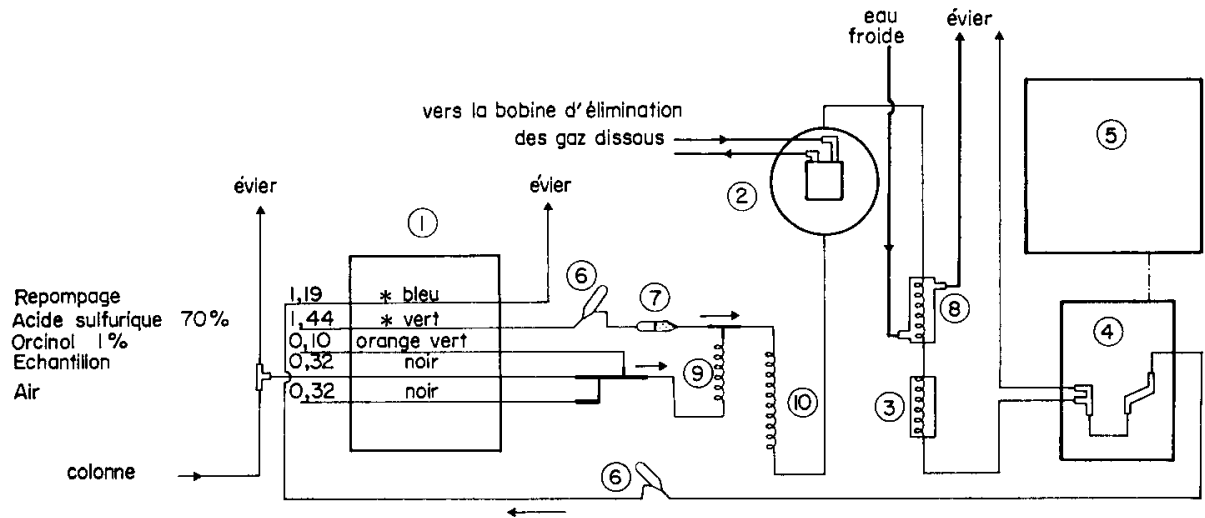

FIG. 3. - Circuit de dosage en contimu des glucides en sortie de colonne

(1) Pompe péristaltique Technicon

(2) Bain-marie Thermostat à circulation Braun Termomix II ; huile SISS, SI 200 , I00 csk ; serpentin $\varnothing \mathrm{I}, 6 \mathrm{~mm}, 95^{\circ} \mathrm{C}$

(3) Lumière excitatrice au néon Technicon

(4) Colorimètre Technicon ou Vitatron, $420 \mathrm{~nm}$

(5) Enregistreur Technicon ou enregistreur intégrateur Vitatron

(6) Chambre anti-retour " pneumatique " en verre

(7) Filtre en laine de verre

(8) Bobine réfrigérée

(9) Petite bobine de mélange

(ro) Grande bobine de mélange

* Tubes en acidflex

Le coefficient de variation est de I,2 p. Ioo pour une série de 20 dosages successifs de saccharose à $5 \mu \mathrm{g} / \mathrm{ml}$ dans de l'eau, la densité optique moyenne étant de o,244 (appareillage Technicon). La sensibilité varie suivant les sucres pour une même concentration, les pentoses réagissent mieux que les hexoses et que les méthyl pentoses. La loi de Beer Lambert n'est pas respectée à des concentrations inférieures à $2 \mu \mathrm{g} / \mathrm{ml}$ pour la plupart des sucres. Elle ne l'est pas toujours lorsque la densité optique dépasse 0,5 (appareillage Technicon).

\section{f) Évaluation quantitative.}

A l'aide de solutions standard de concentration variable, calculer pour chaque sucre, une constante $\mathrm{K}$ qui correspond à la surface du pic obtenu à partir de I microgramme déposé sur la colonne. Dans un échantillon inconnu, la surface $S$ du pic de chaque glucide est corrigée par rapport à la quantité injectée et par rapport au standard externe puis, est divisée par la constante correspondante pour obtenir la concentration $\mathrm{C}$ de ce sucre.

$$
\mathrm{C}=\frac{\mathrm{S} \times 0, \mathbf{I} \times \mathrm{SE}_{\mathrm{reP}}}{\mathrm{P} i \times \mathrm{SE}_{\mathrm{mes}} \times \mathrm{K}}
$$

C : concentration du glucide dans l'échantillon en $\mu \mathrm{g} / \mathrm{ml}$;

$\mathrm{K}$ : constante pour chaque glucide correspondant à la surface du pic obtenu à partir de I microgramme déposé sur la colonne (tabl. 7);

$\mathrm{S}$ : surface du pic ;

$\mathrm{SE}_{\text {ref }}$ : densité optique de référence du standard externe; 
$\mathrm{SE}_{\mathrm{m} \text { es }}$ : densité optique mesurée du standard externe ;

$\mathrm{P} i$ : poids injecté en $\mathrm{g}$.

Cette formule n'est valable que si la fraction d'effluent passant dans l'autoanalyseur est constante.

g) Mesure de la surface des pics du chromatogramme.

La surface des pics est mesurée soit par la méthode manuelle de triangulation (SPACKMAN, STEIn et Moore, I958), soit par une méthode automatique grâce à un intégrateur électronique du type Vitatron.

\section{JUSTIFICATION DE LA MÉ'THODE}

\section{I. --Séparation des glucides}

a) Élimination des gaz dissous.

L'élimination des gaz dissous dans l'éluant avant son entrée dans la colonne n'a jamais été résolue de façon simple et satisfaisante par les systèmes proposés jusqu'à présent (Moore, Spackman et Stein, I958; Spackman, Stein et Moore, I958; ZERFing et WeEning, I966; OHMs et al., I967). Après 1'essai de divers procédés (élimination préalable des gaz sous-vide dans l'autograd, chauffage à $55^{\circ} \mathrm{C}$ des solutions avant 1'emploi) qui se sont révélés peu efficaces et peu pratiques, le dispositif représenté sur le schéma (fig. I) a été adopté. Il élimine convenablement les gaz dissous en continu.

\section{b) Étude du gradient d'élution.}

Le système de CATRAvas (I966) (gradient A) présente trois inconvénients (fig. 2 a) D'une part, le fructose et l'arabinose sont très mal séparés, d'autre part il apparaît trois pics en fin de chromatogramme qui ne correspondent à aucun sucre. Deux de ceux-ci sont situés au même niveau que le xylose et le glucose, ce qui gêne l'interprétation et l'évaluation quantitative des résultats. Enfin, l'élution est longue car elle demande 7 heures jusqu'au glucose. Nous avons donc essayé d'obtenir un gradient d'élution permettant de mieux séparer le fructose de l'arabinose, ne présentant pas d'artefact et assurant une élution complète en un temps plus court.

En faisant varier le rapport des concentrations en borate et chlorure de sodium du gradient d'élution, les artefacts ont été pratiquement éliminés (fig. 2 a). Il reste cependant deux légers bombements de densité optique 0,005 et 0,0I respectivement au niveau $d u$ xylose et $d u$ glucose qui ne disparaissent pas, même en supprimant complètement le chlorure de sodium.

L,e fructose et l'arabinose sont mieux séparés grâce à la modification de la pente du gradient au moment de la sortie de ces deux sucres ; les courbes de concentration en borate et chlorure de sodium ont été calculées d'après la méthode de PETERsSON et Sober (I959). L,es deux sucres n'ont pu être séparés complètement. Il est probable qu'après renouvellement de la résine, cela soit possible car celle utilisée a perdu beaucoup de son pouvoir séparateur au cours du temps et de son usage continu (fig. 2 a chromatogrammes I et 3). Enfin, l'étude de l'influence de la concentration en $\mathrm{NaCl}$ 
(de o à $0,2 \mathrm{M}$ ) a permis d'accélérer l'élution et d'obtenir des pics plus symétriques.

Afin de vérifier que le gradient réel correspond bien à celui calculé, le borate a été dosé en continu à l'entrée et à la sortie de la colonne à l'aide de la réaction à l'acide carminique en milieu sulfurique (CALliCOAT et Wolszon, I959). Dans ce but, un montage permettant le dosage à l'autoanalyseur a été mis au point (fig. 4). La courbe obtenue pour l'éluant est parallèle à la courbe calculée alors que celle correspondant à l'éluat contient plusieurs changements de pente (fig. 5), permettant de diviser les glucides en trois groupes suivant leur affinité pour la résine (KESSLER, I967) : un premier groupe allant du saccharose au cellobiose (voir tableau 8 indiquant l'ordre d'apparition des glucides) est élué jusqu'au premier changement de pente, puis après un certain temps, un deuxième groupe (maltose à ribose) apparaît au changement de pente suivant, enfin le groupe allant du mannose au glucose sort en dernier.

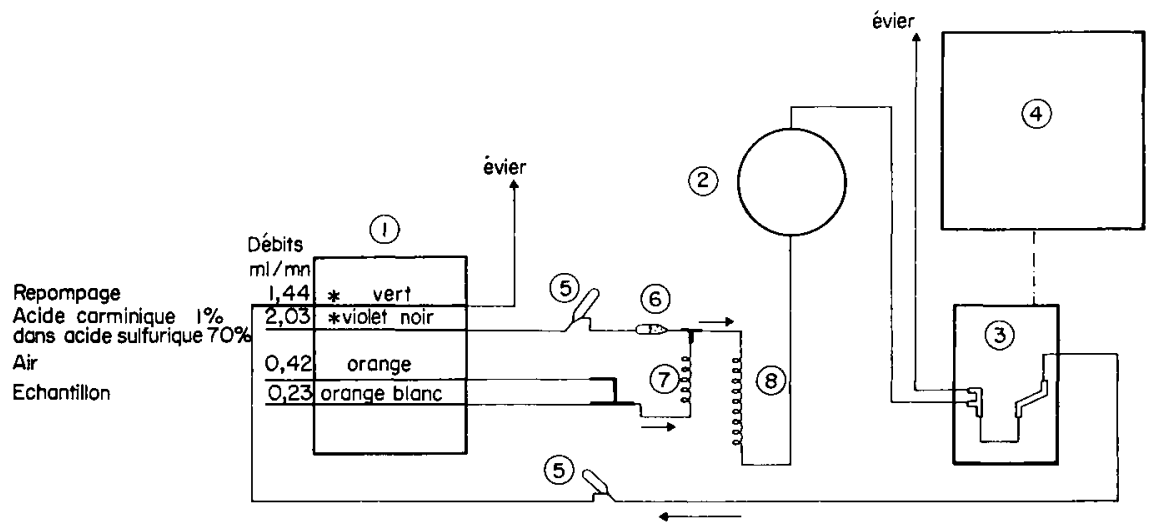

FIG. 4. - Circuit de dosage en continu des borates

(I) Pompe péristaltique

(2) Bain-marie : serpentin $\varnothing \mathrm{I}, 6 \mathrm{~mm}, 30^{\circ} \mathrm{C}$

(3) Colorimètre $625 \mathrm{~nm}$

(4) Enregistreur

(5) Chambre antiretour "pneumatique " en verre

(6) Filtre en laine de verre

(7) Petite bobine de mélange

(8) Grande bobine de mélange

* Tubes en acidflex

c) Influence $d u p H$.

Les réactions entre glucides et borates (BOESEKEN, I949), l'influence du pH sur la formation des composés ionisés (ISBELL et al., I948), la structure des solutions de borate (LoURIJSEN-TEYSSEDRE, I955) et l'affinité des borates pour la résine (EvEREST et POPIEL, I956) nous ont conduit à augmenter l'alcalinité des tampons afin d'améliorer la séparation. Effectivement la résolution du couple fructose-arabinose s'améliore lorsque le $\mathrm{pH}$ croît de 8,0 à Io,o et le temps d'élution diminue (fig. 6), contrairement à ce qu'affirme GREEN (Ig66). Il suffit que 1'alcalinité soit plus élevée en chambre 3 de l'autograd pour obtenir une séparation nettement meilleure avec le gradient $\mathrm{A}$; la résolution obtenue avec le gradient $\mathrm{B}$ est due beaucoup au gradient de $\mathrm{pH}$. Cependant, il faut craindre des réactions secondaires importantes puisque des phénomènes de transformation (LOBRY de BRUYN et AlBERDA VAN EKENSTEIN, I895; Speck, I958) et de dégradation des glucides (Buhler, Thomas et Wang, I954; 
BUHLER et al., 1955) ont lieu en milieu basique. Le $\mathrm{pH}$ optimal adopté est de 9,5 .

La mesure du pH en continu (fig. 5) à l'entrée et à la sortie de la colonne a montré dans l'effluent des irrégularités correspondant aux trois groupes d'oses et d'oligosides.

d) Infuence du débit.

L'examen de la figure 7 montre qu'à partir d'un débit de $\mathrm{I}, 5 \mathrm{ml} / \mathrm{mn}$, la séparation est mauvaise, en revanche, lorsqu'il est égal à $0,25 \mathrm{~m} 1 / \mathrm{mn}$, les pics sont mieux différenciés mais ils traînent davantage. Le volume d'éluant nécessaire à la séparation est le même quelle que soit la vitesse d'écoulement. Douze sucres sont analysés en $2 \mathrm{~h}$ à un débit de $\mathrm{I} \mathrm{ml} / \mathrm{mn}$ avec le gradient $\mathrm{D}$ alors qu'il faut $\mathrm{I}_{5} \mathrm{~h}$ à $0,25 \mathrm{ml} / \mathrm{mn}$ avec le gradient $B$.

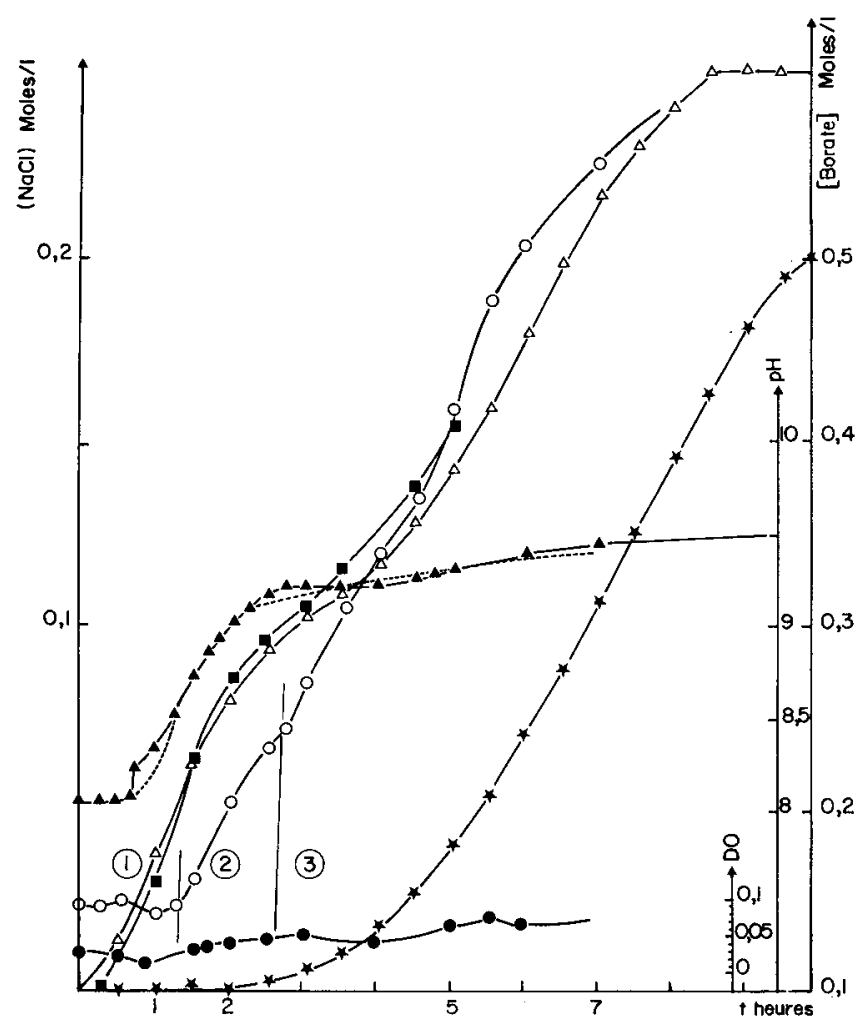

FIG. 5. - Comparaison des gradients de Borate, $\mathrm{NaCl}, \mathrm{pH}$ dans le cas du gradient $B$

$\triangle$ Gradient de borate de sodium calculé

- Gradient de borate de sodium mesuré à l'entrée de la colonne

- Gradient de borate de sodium mesuré en sortie de colonne

* Gradient de $\mathrm{NaCl}$ calculé

-- pH mesuré à l'entrée de la colonne

- pH mesuré en sortie de colonne

- Ligne de base produite par l'éluant traversant seul la colonne

(1) (2) (3) Groupes de glucides par affinité croissante pour la résine 


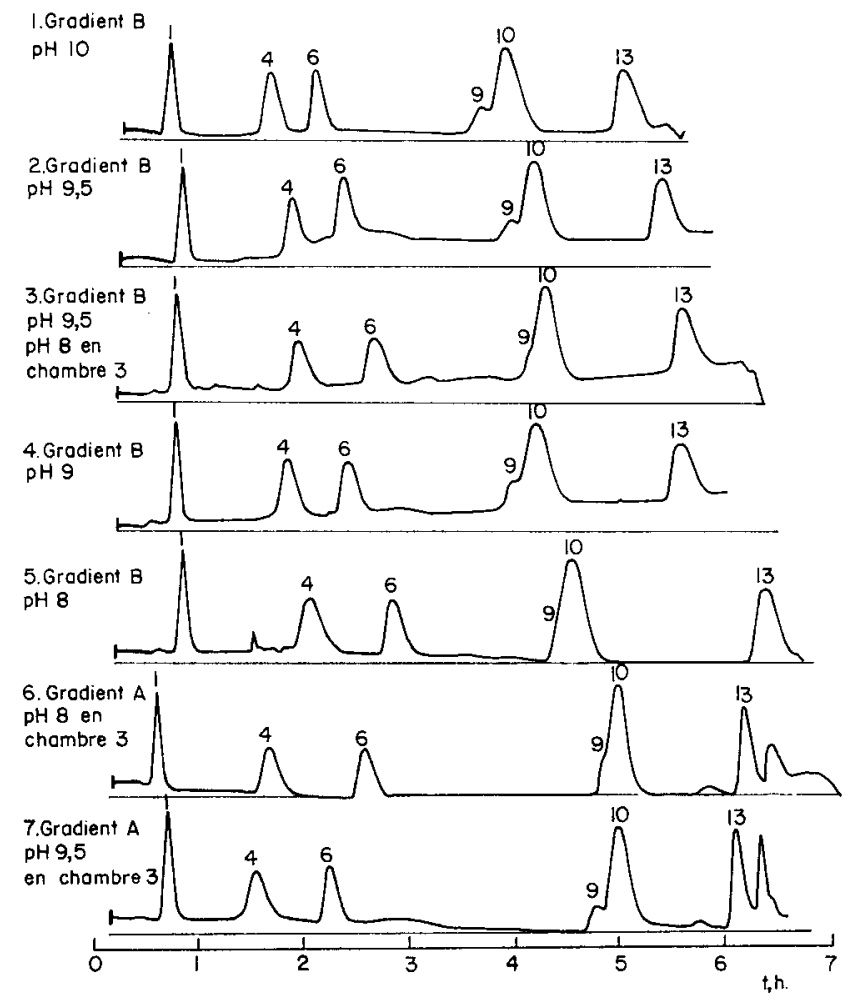

FIG. 6. - Infuence du $p H$

Dépôt de o,I $\mathrm{ml}$ d'une solution contenant $200 \mu \mathrm{g} / \mathrm{ml}$ de chaque glucide. Chromatogramme I à $5:$ le $\mathrm{pH}$ indiqué correspond à celui de la solution la plus alcaline, le tampon de $\mathrm{pH} 8$ étant toujours utilisé dans les chambres indiquées tableau 2.

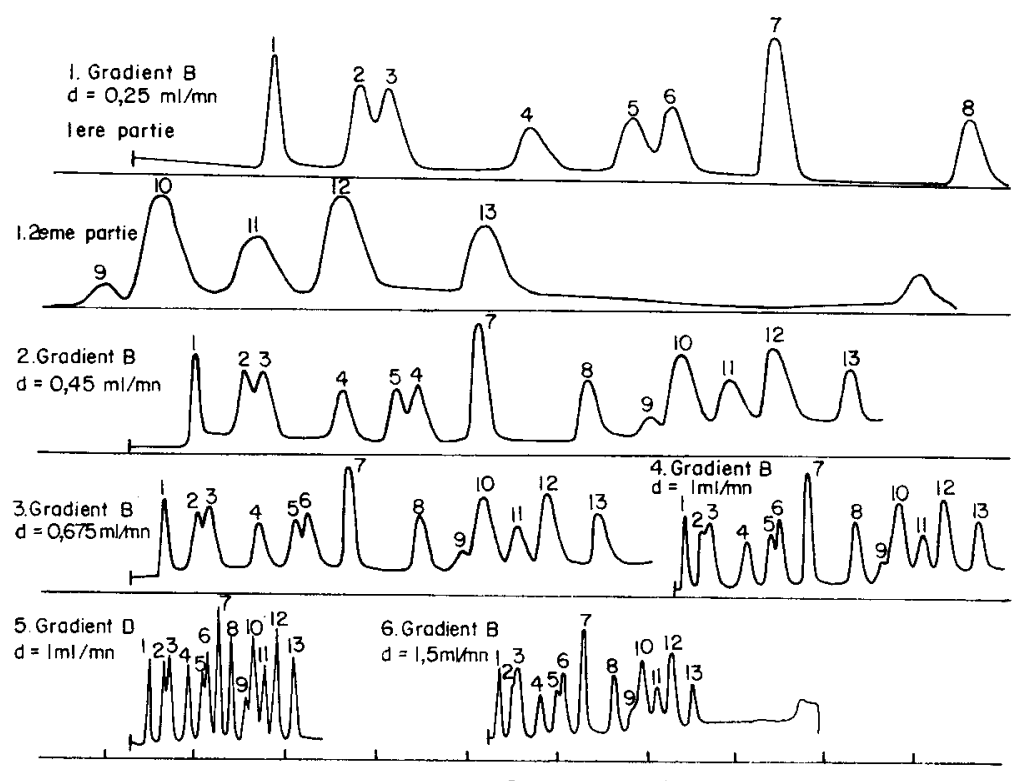

Fig. 7. - Influence du débit

Dépôt de o,r ml d'une solution contenant $200 \mu \mathrm{g} / \mathrm{ml}$ de chaque glucide, le chromatogramme I a dû être coupé en deux parties

Annales de Biologie animale. - 1974. 
e) Influence de la température.

Lorsque la température s'élève de 30 à $65^{\circ} \mathrm{C}$ (fig. 8), la pression diminue et le volume d'élution croît (ARwidi et SAMUEison, I964 et r965 qui éluent les sucres avec un mélange éthanol-eau ont observé le contraire). La séparation s'améliore jusqu'à $60^{\circ} \mathrm{C}$ mais pas au-delà, le couple cellobiose-raffinose est d'autant mieux séparé que la température augmente. Les destructions de glucides augmentent fortement au-dessus de $55^{\circ} \mathrm{C}$. Certains d'entre eux semblent plus thermolabiles que d'autres : cellobiose, maltose, lactose, rhamnose, mannose. Pour cette raison, la température optimale utilisée est de $55^{\circ} \mathrm{C}$.

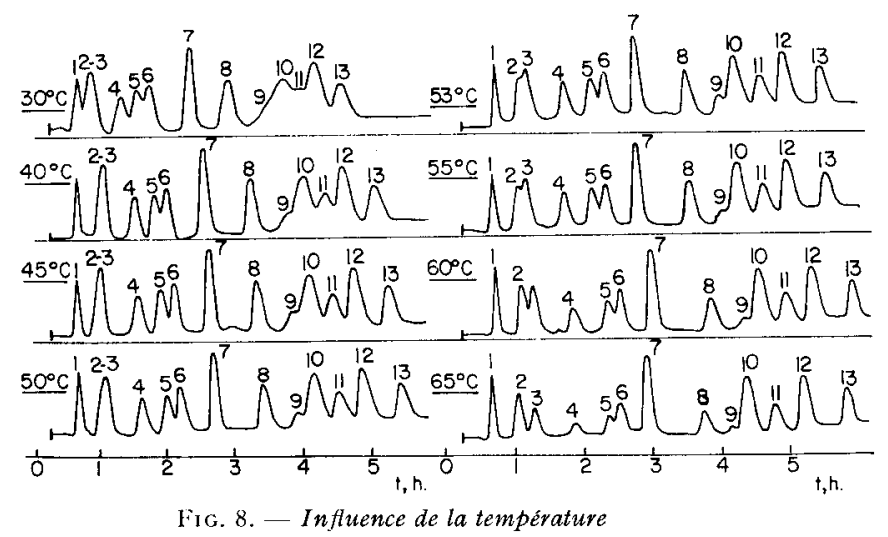

L.es sucres ont été élués par le gradient B.

Un volume de $0,1 \mathrm{ml}$ d'une solution contenant $200 \mu \mathrm{g} / \mathrm{ml}$ de chaque glucide a été déposé dans tous les cas

\section{2. - Dosage des glucides}

a) Circuit du dosage des sucres en sortie de colonne.

Dans le montage réalisé pour le dosage en continu, la solution d'orcinol et celle d'acide sulfurique sont séparées (CATRAVAs, I966) afin d'obtenir une ligne de base régulière et une meilleure conservation des réactifs. Dans ces conditions, il n'est pas nécessaire de recristalliser l'orcinol utilisé. Sur le circuit de dosage, sont disposées deux chambres anti-retour pneumatique (fig. 3) ainsi qu'un filtre en laine de verre qui arrête les particules des tubes de pompage en acidflex entraînées par l'acide. De cette manière, la ligne de base est excellente.

L'influence de la concentration en acide sulfurique sur la sensibilité de la réaction a été étudiée pour différents sucres. Les maxima d'absorption sont différents suivant les oses; une concentration de $70 \mathrm{p}$. Ioo $\mathrm{v} / \mathrm{v}$ correspond à un optimum de la coloration pour la plupart des glucides. Pour un temps de chauffage du mélange réactionnel de $12 \mathrm{mn}$, la sensibilité est doublée lorsque la température du bain-marie passe de 75 à $95^{\circ} \mathrm{C}$. C'est cette dernière température que nous utilisons. De plus, l'emploi de la lumière excitatrice sur le montage (fig. 3) améliore encore la sensibilité. Lorsqu'on augmente le temps de chauffage au-delà de $\mathrm{I} 2 \mathrm{mn}$, la coloration brun jaune ne s'accroît plus guère. Ces résultats rejoignent ceux de BRUCKNER (I955) et de Schonenberger et al. (I957). Il faut un temps de chauffage suffisant pour que la 
TABLEAU 3

Produits interférents dans la réaction à l'orcinol sulfurique

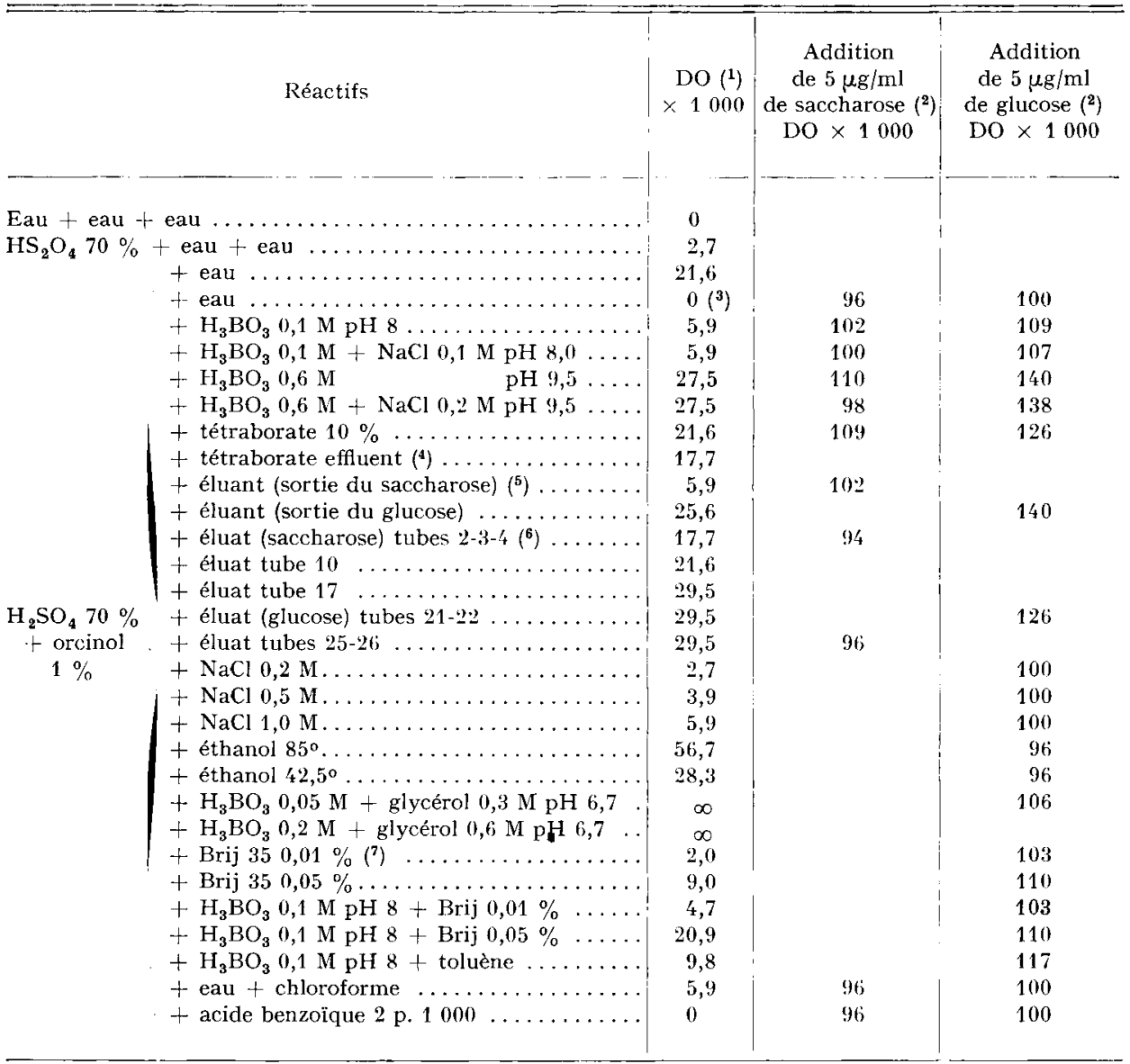

(1) Les valeurs indiquées sont rapportées à une densité optique de 0,1 pour $5 \mu \mathrm{g} / \mathrm{ml}$ de glucose.

(2) Les sucres sont dissous dans la solution dont on étudie l'influence sur la réaction. Les valeurs sont indiquées par rapport à la ligne de base des réactifs.

( $\left.{ }^{3}\right)$ Les valeurs ci-dessous sont indiquées par rapport à la ligne de base acide-orcinol-eau.

(4) Effluent de colonne en fin de régénération.

${ }^{(5)}$ Cette solution a la même composition en borate, $\mathrm{NaCl}$, soude que l'éluat au moment de la sortie du sucre.

${ }_{\left({ }^{6}\right)}$ Il s'agit de l'effluent de colonne recueilli grâce à une fractionneuse lors d'une chromatographie à blanc (un tube toutes les $15 \mathrm{mn}$, gradient $\mathrm{B}$ ). Certains tubes correspondent à la sortie des sucres.

(7) Cette solution est préparée avec $0,1 \mathrm{ml}$ de Brij 35 saturé, pour $1000 \mathrm{ml}$. 
coloration obtenue provienne uniquement de la réaction de l'orcinol avec les dérivés du furfural et non avec d'autres produits de décomposition des glucides (MENDEL, Kemp et MYers, I954). Dans ces conditions, la densité optique est mesurée à $420 \mathrm{~nm}$, ce qui correspond à l'absorbance maximale de la plupart des oses (BRUCKNER, I955 ; SchONENBERGER et al., I957; SchUltze, Schmidtberger et HAUPT, 1958).

\section{b) Produits interférents.}

L'influence des borates sur la réaction glucides-acide sulfurique-chromogène est très controversée (Gregory, I960 ; Françors, Marshali, et Neuberger, I962 ; I in et Pomeranz, ig68). De nombreux produits agissent sur la réaction : le chlorure de sodium, l'ergothionine, le glutathion, le tryptophane, la méthionine, la tyrosine (Mendei, Kemp et Myers, I954), les acides uroniques, les sels de plomb, les nitrates (Brown, I946), le benzaldéhyde, le formaldéhyde ('Tildmans et Philippi, I929). Parmi les produits sans action sur la réaction, notons le chlorure de potassium (GREGORY, I960) et le fluorure de sodium (Mendei,, Kemp et Myers, I954).

Les résultats sur le glucose et le saccharose indiqués au tableau 3 montrent que les borates de sodium et de potassium augmentent légèrement la sensibilité. Le chlorure de sodium réagit peu avec l'orcinol sulfurique et la sensibilité n'est pas modifiée en présence de glucides.

Si le toluène entraîne une légère augmentation de la sensibilité, le chloroforme et l'acide benzoïque n'ont pas d'action ; ces trois produits peuvent donc être utilisés comme conservateurs de solutions standard. Par contre, l'éthanol à $80 \mathrm{p}$. Ioo, le glycérol $0,3 \mathrm{M}$ réagissent fortement et atténuent la coloration donnée par les sucres. Le Brij o,oI p. Ioo réagit avec l'orcinol sulfurique, en sa présence la sensibilité de la réaction avec les glucides est augmentée ; il peut être ajouté sans inconvénient aux tampons d'élution. Enfin, la coloration obtenue avec les glucides est moindre dans l'effluent de colonne que dans une solution de composition identique en borate, chlorure de sodium et de même alcalinité. Sans doute y a-t-il un produit de dégradation de la résine qui influe sur la réaction. La différence est d'environ ro p. Ioo ; ceci est très important pour la mesure des taux de recouvrement des oses.

\section{VALIDITÉ E'T LIMITE DE LA MÉTHODE}

\section{I. - Récupération des glucides}

\section{a) Introduction.}

Sous l'influence de l'alcalinité du milieu et de la température, les glucides subissent certaines transformations (LOBRy de BRUYN et AlBERda VAN EKENs'teIn, I895; Hough, JoNes et Richard, I953, I954 ; Speck, I958), la principale étant l'épimérisation. Ils peuvent aussi être hydrolysés dans le cas de diholosides (REBENFELD et PAcsu, 1953) et dégradés en différents acides sous l'effet catalytique d'une résine échangeuse d'anions, forme $\mathrm{OH}^{(-)}$(Philitips et Pollard, I953; Hulme, I953; Buhler, Thomas et Wang, I954; Bul,Her et al., I955). Si la présence de borates augmente la transformation du glucose en fructose, elle diminue par contre la trans- 
formation inverse et la destruction de ces deux sucres (MENDicino, I960). CARUBELLI (r966) a montré que le maltose, le lactose et le cellobiose élués par des tampons borates alcalins se transforment dans la résine à température ambiante et donnent lieu à plusieurs pics. FLORIDI (I97I) note la transformation du lactose en un autre produit dans des conditions comparables.

Les résultats des différents auteurs concernant les taux de récupération des glucides séparés sur résine échangeuse d'anions par des tampons borate sont très variables, (Hallen, ig6o; Syamananda, Staples et Block, I962; Walborg, ChrisTENSSON et GARDELL, I965; OhMs et al., I967 ; Jolley et FreEMAN, I968 ; Mundie, Cheshire et BACON, I968; LEE, MCKELVy et LANG, I969). L'évaluation du tatux de récupération de $\mathbf{2} 2$ sucres a été faite à concentration élevée de façon à se placer dans le cas le plus défavorable. Deux méthodes chimiques et une méthode radiochimique ont été utilisées.

\section{b) Évaluation par voie chimique}

La première méthode consiste à comparer la surface obtenue après chromatographie à celle donnée par une même quantité de glucide prélevée pendant ro mn et dosée par l'autoanalyseur. Ce sucre est dissous dans la fraction d'effluent de la colonne correspondant à sa sortie, recueillie pendant une chromatographie à blanc (aucun sucre n'a été déposé sur la colonne). Un standard externe de saccharose à $5 \mu \mathrm{g} / \mathrm{ml}$ permet de tenir compte des variations dues à l'appareil de dosage. Pour cette étude, nous avons utilisé un colorimètre et un enregistreur Technicon. La surface des pics a été déterminée manuellement par la méthode des trapèzes (monographie Technicon $n^{\circ} \mathrm{I}, \mathrm{I}$ 966) qui est plus précise que la méthode du triangle car les pics ne sont pas tous symétriques. Les taux de récupération ont été évalués de cette manière pour I I sucres.

La deuxième méthode consiste à recueillir chaque glucide en sortie de colonne dans un volume connu et à évaluer sa concentration par rapport à une gamme éta!on de ce même sucre préparée dans la fraction d'éluat correspondant à sa sortie de la colonne. Un volume suffisant de cette fraction doit être recueilli lors de nombreuses chromatographies à blanc. On compare ensuite la quantité obtenue à celle déposée sur la colonne. Cette méthode, plus fastidieuse que la première, a été utilisée pour 7 sucres afin de vérifier les résultats obtenus précédemment.

\section{c) Évaluation par voie radiochimique.}

Les taux de récupération radiochimique du glucose et du fructose uniformément marqués au ${ }^{14} \mathrm{C}$ ont été évalués ; ces derniers sont entraînés par les mêmes sucres non radioactifs. Cette méthode est beaucoup plus sensible et précise que les deux précédentes. La radioactivité est mesurée au moyen d'un compteur à scintillation liquide Packard Tricarb $3375\left({ }^{9}\right)$. La fraction d'effluent de la colonne qui n'est pas envoyée dans l'autoanalyseur est recueillie par fractions de $5 \mathrm{mn}$ grâce à un collecteur de fractions. On rajoute ro $\mathrm{ml}$ d'instagel et une quantité d'eau suffisante pour avoir un gel stable. En effet, entre I 2 et $24 \mathrm{p}$. Ioo de phase aqueuse dans l'émulsifiant, le gel est instable et des sels précipitant dans la plupart des tubes risquent d'entraîner des 
produits radioactifs. Un témoin contenant un poids connu de la solution à analyser est préparé dans les mêmes conditions. On compare la radioactivité des tubes à celle injectée en tenant compte de la fraction de volume d'effluent qui n'est pas recueillie. La perte de rendement due au quenching est évaluée par l'A. E. S. (Automatic External Standardisation).

L'activité spécifique des sucres marqués étant très élevée, la quantité analysée est très faible ; aussi, elle ne pourrait être appréciée par le dosage colorimétrique. Pour parvenir malgré tout à comparer le radiochromatogramme à son homologue colorimétrique et afin d'éviter une traînée radiochimique, chaque sucre marqué est additionné d'une quantité appréciable de ce même glucide non radioactif.

\section{d) Résultats.}

Les résultats obtenus (tabl. 4) sont semblables avec les trois procédés sauf dans le cas du mannose. Ils sont comparables à ceux de WALBORG, RAY et OHRBERG (I969). Les sucres dont le taux de récupération est le plus faible sont les mêmes : maltose et mannose. Les taux que nous trouvons sont en général moins élevés car ces auteurs utilisent des tampons neutres qui respectent mieux les sucres. La reproductibilité des mesures de récupération est cependant peu différente de celle de ces auteurs, elle varie suivant les sucres. En outre, les différences entre expériences ne vont pas dans le même sens pour tous les sucres. Il est donc illusoire d'ajouter un standard interne qui n'évoluerait pas de la même manière que certains glucides. Par contre, nous utilisons un standard externe car la sensibilité de la réaction varie à chaque fois, principalement à cause du vieillissement des réactifs et de l'usure des tubes du montage analytique.

Les radiochromatogrammes montrent que le pourcentage de glucose transformé (tabl. 4) est inférieur au taux d'impuretés radiochimiques qui est de 2 p. roo. Le dosage colorimétrique de l'effluent ne montre d'ailleurs aucun pic au niveau des sommets inconnus présents sur le radiochromatogramme. En revanche, le taux de transformation du fructose en d'autres glucides est supérieur au taux d'impuretés. Ce sucre semble s'être converti légèrement en glucose, les pics radioactif et colorimétrique donnant une valeur de $\mathrm{I}$ p. roo. Ce glucose peut être à l'état d'impureté dans l'échantillon ou provenir d'une épimérisation du fructose dans la colonne. Il faudra donc être extrêmement prudent lors de l'interprétation d'un chromatogramme présentant du fructose.

L'élution de solutions ne contenant qu'un seul sucre étalon nous a permis d'évaluer ses transformations possibles. Le saccharose ne donne aucun autre glucide détectable, le cellobiose qui a un taux de recouvrement de $85,5 \mathrm{p}$. roo donne des traces d'un autre diholoside, de fructose et de glucose.

Il semble donc d'après le tableau 4, qu'en cours de chromatographie, les sucres ne se transforment les uns dans les autres que de façon négligeable, mais qu'ils se dégradent considérablement à un taux constant pour la concentration étudiée. Il convient de temps en temps de procéder à la purification de la résine telle qu'elle est décrite dans le mode opératoire. En effet, l'acide chlorhydrique élimine les éventuels acides issus de la dégradation des glucides qui seraient restés fixés, la soude entraîne les silicates qui proviendraient de l'attaque du verre par les tampons à forte concentration en borate. 


\section{TABLEAU 4}

Taux de récupération et de transformation des glucides

Méthode I : comparaison de surfaces

Méthode 2 : évaluation de la quantité de sucre en sortie de colonne

$\mathrm{S}$ : écart-type de la population

\begin{tabular}{|c|c|c|c|c|c|c|c|c|c|c|}
\hline \multirow{2}{*}{ Glucides } & \multicolumn{3}{|c|}{$\begin{array}{l}\text { Méthode chimique } \\
n^{\circ} 1\end{array}$} & \multicolumn{3}{|c|}{$\begin{array}{l}\text { Méthode chimique } \\
n^{0} 2\end{array}$} & \multicolumn{3}{|c|}{$\begin{array}{l}\text { Méthode radio- } \\
\text { chimique }\end{array}$} & \multirow{2}{*}{$\begin{array}{l}\text { Pourcentage } \\
\text { de glucide } \\
\text { transformé }\end{array}$} \\
\hline & $\begin{array}{l}\text { P. } 100 \\
\text { recoul- } \\
\text { vré }\end{array}$ & $\begin{array}{l}\text { Nbre } \\
\text { exp. }\end{array}$ & $\pm \mathrm{S}$ & $\begin{array}{l}\text { P. } 100 \\
\text { recou- } \\
\text { vré }\end{array}$ & $\begin{array}{l}\text { Nbre } \\
\text { exp. }\end{array}$ & $\pm \mathrm{s}$ & $\begin{array}{c}\text { P. } 100 \\
\text { recou- } \\
\text { ré }\end{array}$ & $\begin{array}{l}\text { Nbre } \\
\text { exp. }\end{array}$ & $\pm \mathrm{s}$ & \\
\hline Saccharose & 98,0 & 5 & 3,7 & 100,6 & 4 & 3,5 & & & & Traces \\
\hline Raffinose & $9 / 4,6$ & 5 & 4,8 & & & & & & & \\
\hline Cellobiose & 85,5 & 2 & 2,1 & 86,8 & 4 & 4,9 & 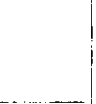 & & & $\left.\begin{array}{l}\text { Diholoside } \\
\text { Fructose } \\
\text { Glucose }\end{array}\right\}$ Traces \\
\hline Maltose & 71,6 & 5 & 2,4 & 75,2 & 4 & 3,1 & & & & \\
\hline Lactose & 85,4 & 5 & 5,7 & & & & & & & \\
\hline Rhamnose & & & & 76,4 & 4 & 3,6 & & & & \\
\hline Ribose & 94,3 & 5 & 2,5 & & & & & & & \\
\hline Mannose & 73,1 & 5 & 3,2 & 66,3 & 4 & 3,3 & & & & \\
\hline Fructose & 76,7 & 4 & 2,2 & 77,2 & 4 & 3,3 & & & & Glucose $1,3 \%$ \\
\hline $\begin{array}{l}\text { Fructose }{ }^{14} \mathrm{C}(\mathrm{U}) \\
(+ \text { fructose })\end{array}$ & & & & & & & 78,4 & 1 & & $\begin{array}{l}\text { Inconnu } 1: 0,4 \% \\
\text { Mannose }: 0,8 \% \\
\text { Inconnu } 2: 2,3 \% \\
\text { Glucose }: 1 \% \%\end{array}$ \\
\hline Galactose & 96,0 & 5 & 6,3 & & & & & & & \\
\hline Xylose & 99,6 & 5 & 4,3 & 98,6 & 4 & 5,9 & & & & \\
\hline Glucose & 87,1 & 1 & & & & & & & & Traces \\
\hline $\begin{array}{l}\text { Glucose }{ }^{14} \mathrm{C}(\mathrm{U}) \\
(+ \text { glucose })\end{array}$ & & & & & & & 86,6 & $\stackrel{2}{2}$ & 1,7 & $\begin{array}{l}\text { Fructose } 1,1 \% \\
\text { Inconnu } 0,5 \%\end{array}$ \\
\hline
\end{tabular}




\section{2. - Évaluation quantitative}

a) Précision en fonction de la concentration et de la méthode d'évaluation de la surface.

Les glucides en solutions standards ont été chromatographiés plusieurs fois de suite à différentes concentrations (tab1. 5). Les deux méthodes précitées d'évaluation de la surface des pics ont été comparées. L'examen du tableau 6 montre que lorsque la concentration est faible (série I), le coefficient de variation est plus élevé pour les deux méthodes. En outre, la triangulation donne une erreur significativement plus faible pour l'ensemble des données ; elle est surtout préférable à la méthode par intégration électronique pour les faibles concentrations, n'étant pas significativement différente de cette dernière pour les séries $2,3,4,5$. Il conviendra donc de travailler autant que possible dans une gamme de concentration suffisamment élevée.

TABLEAU 5

Concentrations des glucides étudiés

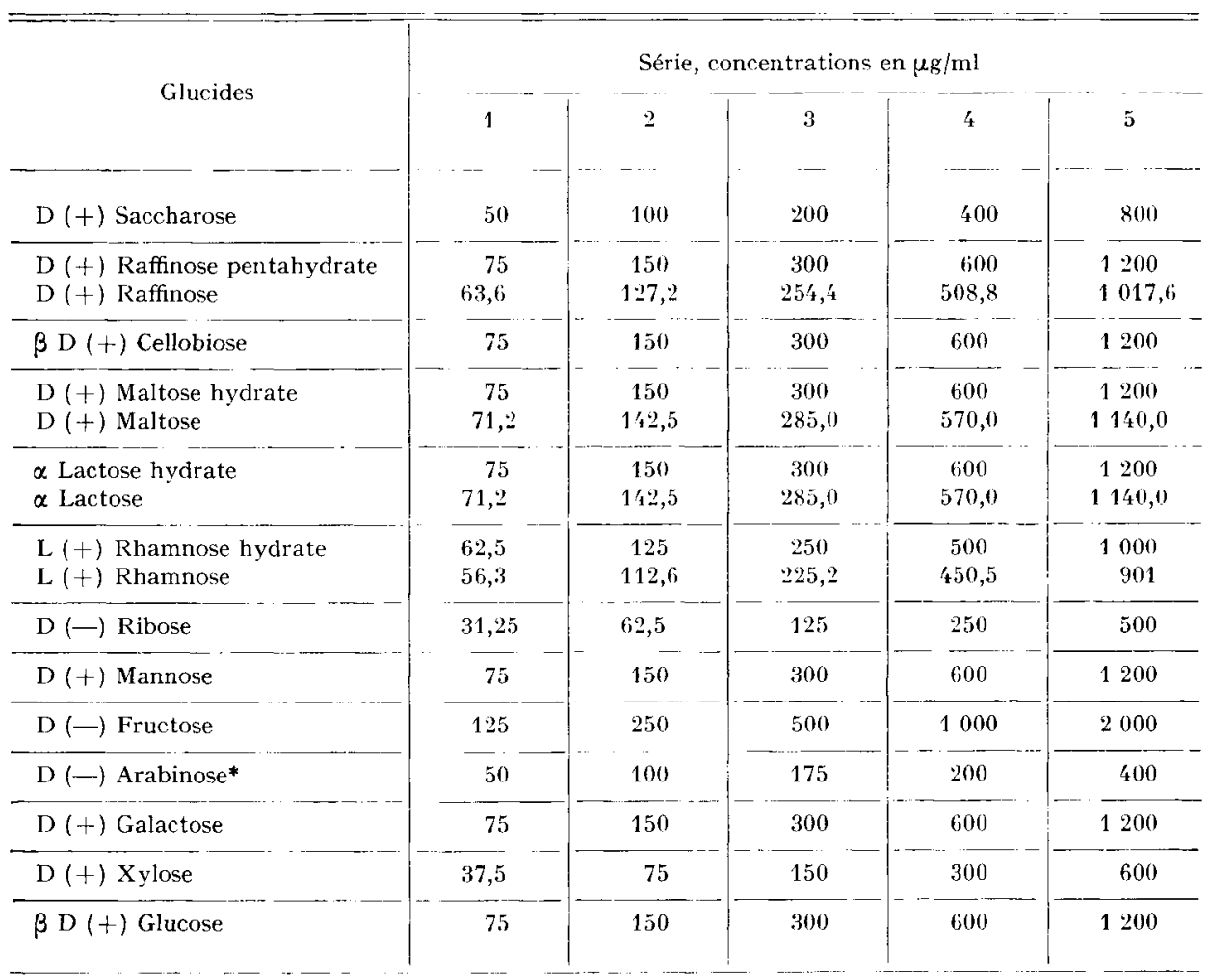

* Nous avons procédé à trois déterminations par série pour tous les sucres indiqués sauf pour l'arabinose. Le nombre d'expériences concernant ce sucre pour les séries $1,2,3$, 4 et 5 est respectivement le suivant $: 1,2,3,4,2$. 
b) Détermination de la constante de proportionnalité.

Nous avons déterminé la constante $\mathrm{K}$ pour chaque sucre avec deux méthodes d'intégration et son intervalle de confiance suivant la concentration (tabl. 7). Il y a une bonne proportionnalité surface-concentration en glucide (fig. 9) sauf pour les faibles concentrations de certains d'entre eux. La constante est alors différente. Le rapport $\frac{\mathrm{K}_{\mathrm{T}}}{\mathrm{K}_{\mathrm{T}}}$ varie beaucoup lorsque les pics sont dissymétriques (glucose). Dans ce cas, les valeurs données par triangulation sont moins exactes.

TABLEAU 6

Précision de l'évaluation de la surface des pics par la méthode de triangulation et pav la méthode d'intégration

\begin{tabular}{|c|c|c|c|c|c|}
\hline \multirow[b]{2}{*}{ Concentrations } & \multicolumn{2}{|c|}{ Triangulation } & \multicolumn{2}{|c|}{ Intégration } & \multirow{2}{*}{$* t$} \\
\hline & $\begin{array}{c}\text { Nbre de } \\
\text { données (1) }\end{array}$ & Moyenne & $\begin{array}{c}\text { Nbre de } \\
\text { données (1) }\end{array}$ & Moyenne & \\
\hline Série 1 & 12 & 3,083 & 12 & 5,508 & $2,794^{*}$ \\
\hline Série 2 & 12 & 1,942 & 12 & 2,608 & 1,951 \\
\hline Série $3 \ldots$ & 12 & 2,100 & 12 & 2,017 & 0,360 \\
\hline Série $4 \ldots$ & 12 & 1,661 & 12 & 1,900 & 1,539 \\
\hline Série $5 \ldots$ & 12 & 1,708 & 12 & 1,108 & 1,918 \\
\hline
\end{tabular}

Anayse de la variance

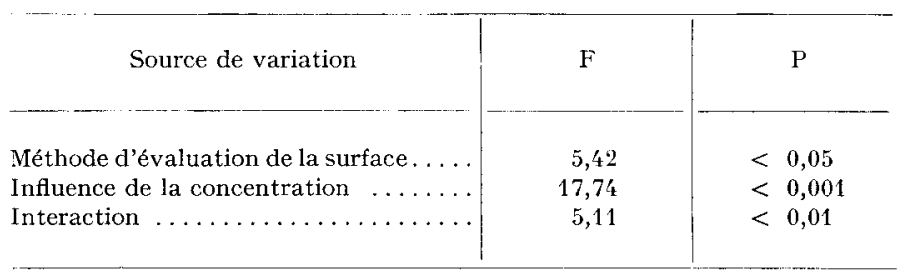

(1) Chaque donnée est le coefficient de variation de trois mesures faites à concentration identique pour un même sucre, la surface des pics de $12 \mathrm{glu}$ cides ayant été évaluée.

${ }^{*} t$ : test $t ; *$ désigne les valeurs significativement différentes.

c) Autre méthode d'évaluation.

Il n'est pas possible d'évaluer la quantité de sucre uniquement par la hauteur des pics comme le font certains auteurs (KESLER, I967 ; FLORIDI, I97I) car la largeur à mi-hauteur n'est pas constante lorsque la concentration varie (tabl. 8). En outre, elle évolue dans le temps avec le pouvoir de résolution de la résine. 
d) Validité de la méthode.

Le taux de récupération est constant à une concentration donnée et la reproductibilité de l'évaluation quantitative est satisfaisante sur toute une gamme de concentration. Ceci montre que le taux de récupération des glucides est constant quelle que soit la concentration. Les réactions de décomposition des glucides ne sont donc pas gênantes comme nous l'avions craint.

\section{TABLEAU 7}

Constantes d'évaluation quantitative pour différents glucides et deux méthodes d'évaluation de la surface

Toutes ces valeurs sont exprimées pour les glucides anhydres $K_{\mathrm{T}}$ : constantes lorsque la surface est évaluée par triangulation $K_{I}$ : constantes lorsque la surface est évaluée par intégration $\mathrm{I}_{\mathrm{C}}$ : intervalle de confiance de la moyenne en $\mathrm{p}$. Ioo.

\begin{tabular}{|c|c|c|c|c|c|c|c|}
\hline \multirow{2}{*}{$\begin{array}{l}\text { Glucides } \\
\text { anhydres }\end{array}$} & \multirow{2}{*}{$\begin{array}{c}\text { Concentration } \\
\mu \mathrm{g} / \mathrm{ml}\end{array}$} & \multirow{2}{*}{$\begin{array}{l}\text { Nbre de } \\
\text { données }\end{array}$} & \multicolumn{2}{|c|}{ Triangulation } & \multicolumn{2}{|c|}{ Intégration } & \multirow{2}{*}{$\frac{\mathrm{K}_{\mathrm{I}}}{\overline{\mathrm{K}_{\mathrm{T}}}}$} \\
\hline & & & $\mathrm{K}_{\mathbf{T}}$ & $I_{C}(\%)$ & $\mathrm{K}_{\mathrm{I}}$ & $I_{C}(\%)$ & \\
\hline Saccharose & 50 à 800 & 15 & $1,0,3$ & 2,2 & 0,51 & 2,7 & 12,65 \\
\hline Raffinose & $\begin{array}{c}6.3,6 \\
127,2 \text { à } 1017,6\end{array}$ & $\begin{array}{r}3 \\
12\end{array}$ & $\begin{array}{l}4,98 \\
5,21\end{array}$ & 0,9 & $\begin{array}{l}0,60 \\
0,65\end{array}$ & 1,2 & $\begin{array}{l}12,05 \\
12,66\end{array}$ \\
\hline Cellobiose & 150) à 1200 & $\begin{array}{r}3 \\
12 \\
\end{array}$ & $\begin{array}{l}4,78 \\
4,37\end{array}$ & 2,4 & $\begin{array}{l}0,61 \\
0,5 \prime\end{array}$ & 2,8 & $\begin{array}{l}12,69 \\
12,35\end{array}$ \\
\hline Maltose & 71,2 à 1140 & 15 & $3,7 x$ & 1,0 & 0,47 & 1,1 & 12,43 \\
\hline Lactose & 71,2 à 1140 & 15 & 4,91 & 0,9 & $0,6 \geq$ & 1,9 & 12,59 \\
\hline Rhamnose & 56,3 à 901 & 15 & 5,28 & 1,4 & 0,67 & 1,8 & 12,68 \\
\hline Ribose & 31,25 à 600 & 15 & 11,81 & 1,2 & 1,69 & 1,0 & 12,64 \\
\hline Mannose & 75 à 1000 & 15 & 5,50 & 1,0 & 0,69 & 2,1 & $12,5_{4}^{\prime}$ \\
\hline Fructose & $\begin{array}{c}125 \\
250 \\
500 \text { à } 2000\end{array}$ & $\begin{array}{l}3 \\
3 \\
9\end{array}$ & $\begin{array}{l}1,81 \\
2,11 \\
3,00 \\
\end{array}$ & 0,1 & $\begin{array}{l}0,21 \\
0,26 \\
0,38\end{array}$ & 0,0 & $\begin{array}{l}11,41 \\
12,32 \\
12,53\end{array}$ \\
\hline Arabinose & 50 à 400 & 12 & 12,57 & 2,9 & & & \\
\hline Galactose & 75 à 1200 & 15 & 5,09 & 1,1 & 0,63 & 2,6 & 12,61 \\
\hline Xylose & 37,5 à 600 & 15 & 11,22 & 2,6 & 1,42 & 3,0 & \\
\hline Glucose & $\begin{array}{c}\quad 75 \\
150 \text { à } 1200\end{array}$ & $\begin{array}{r}3 \\
12\end{array}$ & $\begin{array}{l}6,6^{\prime} \\
5,77\end{array}$ & 0,1 & $\begin{array}{l}0,85 \\
0,75\end{array}$ & 0,8 & $\begin{array}{l}12,80 \\
12,97\end{array}$ \\
\hline
\end{tabular}

\section{3. - Volume de rétention}

Les écarts-types des distances des glucides par rapport à l'origine sont très faibles pour une série de chromatogrammes faits dans les mêmes conditions à des concentrations en sucres différents (tab1. 8). Ils varient de $0,2 \mathrm{~cm}$ pour le rhamnose à $\mathrm{I}, 5 \mathrm{~cm}$ pour le glucose. 


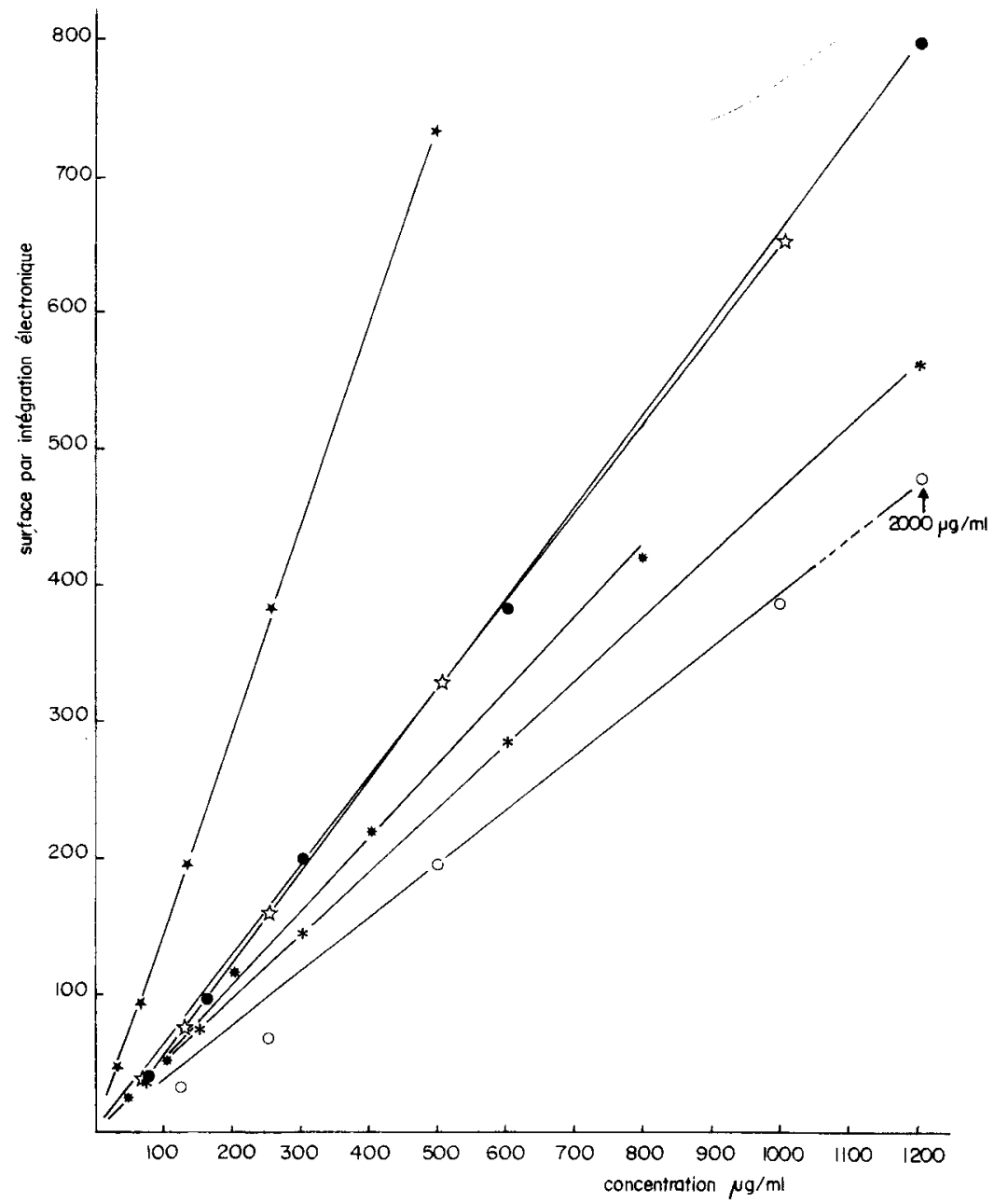

FIG. 9 ( ( ${ }^{\mathrm{re}}$ partie). - Relation entre la surface des pics et la concentration des glucides La surface est évaluée par intégration électronique

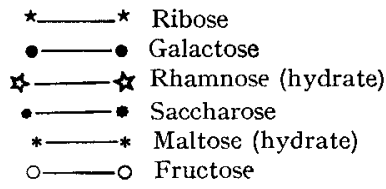




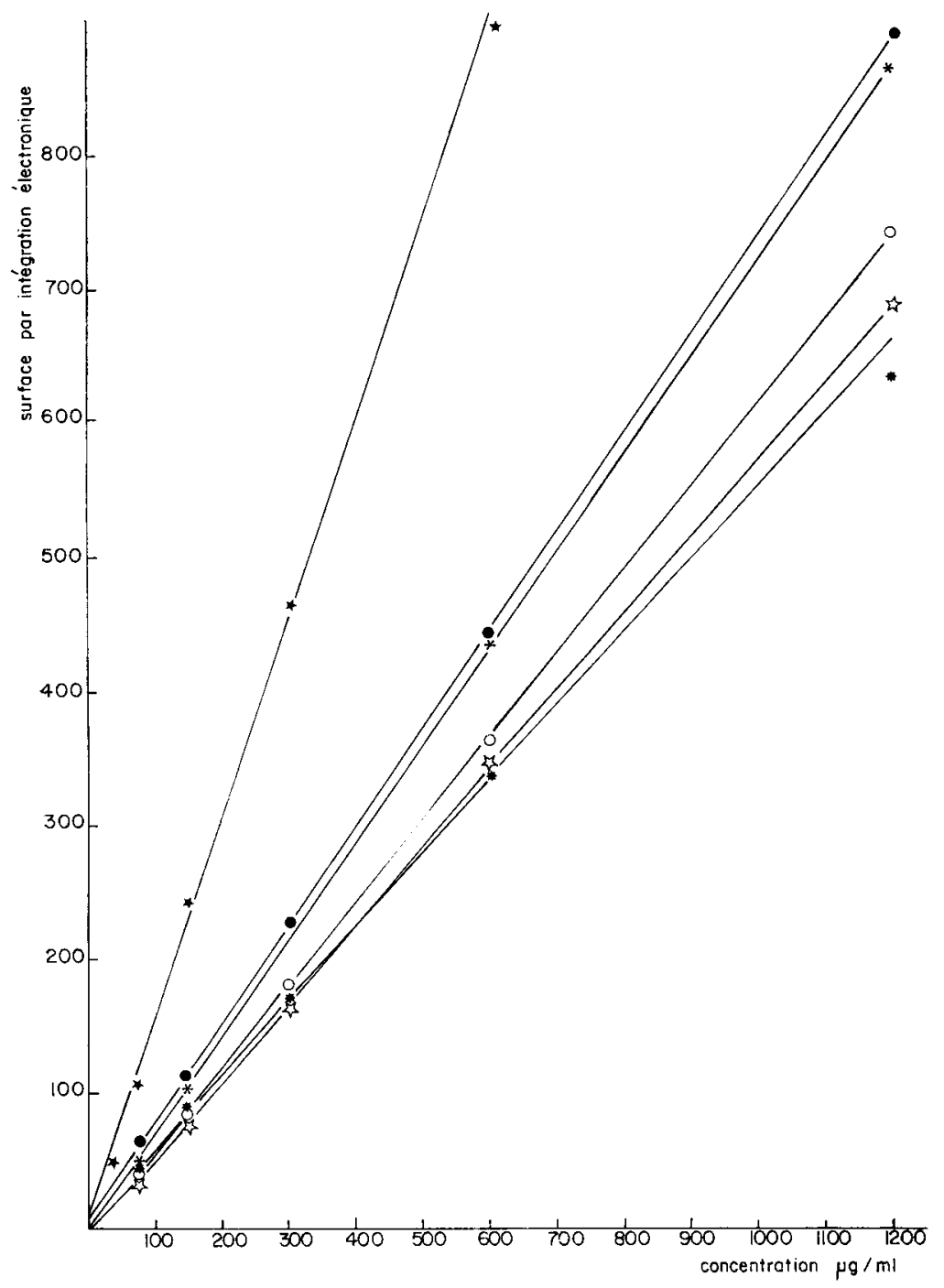

[IG. 9 (2 ${ }^{\mathrm{e}}$ partie)

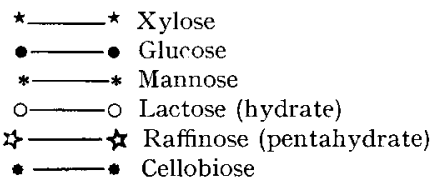




\section{TABLEAU 8}

Ordre d'élution et largeur des pics à mi-hauteur pour divers glucides (Gradient B)

D moy. : distance moyenne des glucides par rapport à l'origine pour diverses concentrations. L'origine correspond au départ du chromatogramme.

$a:$ largeur moyenne des pics à mi-hauteur suivant la concentration des glucides. $\mathrm{S}$ : écart-type.

\begin{tabular}{|c|c|c|c|c|c|c|c|c|}
\hline & \multirow{2}{*}{$\begin{array}{l}\text { Nbre de } \\
\text { domnées }\end{array}$} & \multirow{2}{*}{$\begin{array}{l}\text { D moy } \\
\text { (cm) }\end{array}$} & \multirow{2}{*}{$\begin{array}{l} \pm \mathrm{S} \\
(\mathrm{cm})\end{array}$} & \multicolumn{5}{|c|}{$a(\mathrm{~mm}) 3$ déterminations par série } \\
\hline & & & & 1 & 2 & 3 & 1 & 5 \\
\hline 2-Désoxyribose $\ldots \ldots$ & 2 & 9 & & & & & & \\
\hline Saccharose $\ldots \ldots \ldots$ & 15 & 9,9 & 0,3 & 6 & 6 & 6 & 6,2 & 6,5 \\
\hline Tréhalose $\ldots \ldots \ldots \ldots$ & 2 & 10,6 & & & & & & \\
\hline D-Méthylglucoside . . . & 2 & 12,5 & & & & & & \\
\hline 2-Désoxygalactose ... & 2 & 13,2 & & & & & & \\
\hline Raffinose & 15 & 15,4 & 0,4 & 10 & 11 & 12 & 12,7 & 13,5 \\
\hline Cellobiose & 15 & 17,3 & 0,4 & 11,1 & 10,8 & 11,5 & 12,3 & 12,5 \\
\hline 2-Désoxyglucose $\ldots \ldots$ & $\underline{-}$ & 25,0 & & & & & & \\
\hline Maltose $\ldots \ldots \ldots \ldots$ & 15 & 25,3 & 0,3 & 11,8 & 11,6 & 12,3 & 12,7 & 13,2 \\
\hline Lactose $\ldots \ldots \ldots \ldots$ & 15 & 31,6 & 0,7 & 11,2 & $1 \%$ & $1^{\prime} \mathbf{t}$ & 15,6 & 18,5 \\
\hline Rhamnose ........ & 15 & 33,5 & 0,2 & 9,8 & 9,3 & 10,3 & 11,0 & 11,7 \\
\hline Ribose .......... & 15 & 40,7 & 0,8 & 7,5 & 8,7 & 9,5 & 10,5 & 11,7 \\
\hline Mannose & 15 & 51,1 & 0,8 & 10 & 10 & 10,5 & 11,5 & 12,7 \\
\hline Fructose $\ldots \ldots \ldots$ & 15 & 58,6 & 0,3 & 15,3 & 14,5 & 16,7 & 17,7 & 19,3 \\
\hline Arabinose $\ldots \ldots \ldots$ & 11 & 61,8 & 0,3 & 12 & 12,2 & 12,5 & 12,5 & 13,5 \\
\hline Fucose $\ldots \ldots \ldots \ldots$ & 2 & 64,2 & & & & & & \\
\hline Galactose ........ & 15 & 67,0 & 1,1 & 15 & 16,5 & 18 & 18,7 & 20 \\
\hline Sorbose $\ldots \ldots \ldots$ & 4 & 72 & & & & & & \\
\hline Xylose .......... & 15 & 72,8 & 0,4 & 12,8 & 12,5 & 13,8 & 15,2 & 16,5 \\
\hline Glucose $\ldots \ldots \ldots$ & 15 & 80,4 & 1,5 & 13 & 14,2 & 15,5 & 16,2 & 17,2 \\
\hline
\end{tabular}

\section{CONCLUSION}

Une méthode de séparation des glucides sur résine échangeuse d'anions par un gradient d'élution croissant en borates, en $\mathrm{pH}$ et en chlorure de sodium est étudiée. Après avoir défini les paramètres de l'élution, les conditions optimales de travail sont déterminées ainsi que les marges d'erreur de la méthode du point de vue qualitatif et quantitatif. Le fructose et l'arabinose sont séparés suffisamment pour pouvoir bien les distinguer mais pas assez pour obtenir deux pics indépendants.

Le procédé mis au point convient particulièrement à des mélanges de mono- diet triholosides ; il permet de gagner beaucoup de temps par rapport à d'autres méthodes (l'élution jusqu'au glucose varie entre $2 \mathrm{~h}$ et $5 \mathrm{~h} 30$ suivant le gradient de concentration) ; en outre, il est d'une grande souplesse d'utilisation suivant la composition glucidique des échantillons à analyser. Sa précision est satisfaisante (l'intervalle de confiance sur la moyenne de $\mathrm{K}$ n'est pas supérieur à 3,0 p. Ioo) à condition de prendre certaines précautions et d'analyser des solutions contenant les sucres dans un intervalle de concentration optimal. 
Le dosage colorimétrique des sucres avec l'autoanalyseur a été étudié de façon à obtenir une sensibilité optimale pour une meilleure qualité des résultats. L'influence sur la réaction de divers composés a été évaluée.

La mesure des taux de recouvrement et la chromatographie de glucides étalons a permis de voir que, contrairement à nos craintes, dans ces conditions opératoires, ces derniers ne sont pratiquement pas transformés les uns dans les autres et que, s'ils sont considérablement dégradés, ils le sont à un taux constant. Enfin, la reproductibilité du volume d'élution de chaque glucide est excellente.

Reçu pour publication en janvier 1974.

\title{
SUMMARY
}

\author{
SEPARATION OF OSES, DI AND TRISACCHARIDES \\ BY ION-EXCHANGE CHROMATOGRAPHY
}

We have studied an automatic method of separating and determining mono- di- and trisaccharides of biological origin by chromatography of carbohydrate complexes with borates using resin anion exchange. The sugars are eluted by a gradient of borate, sodium chloride and soda concentration. The operating conditions for good carbohydrate separation are described. A mixture of 13 sugars, mono- di- and triholosides is analyzed in 4 hours (from deposit of the sample to exit of the glucose); a mixture containing fewer carbohydrates of these three classes is analyzed in 2 hours (from deposit of the sample to exit of the glucose) (fig. 2 and 7 ).

The following results were obtained by this method :

I. All phases of manipulation are automatized with the equipment; a simple apparatus continuously and efficiently eliminates gas dissolved in the eluent before it enters the column (fig. I).

2. By determining the method of loading the resin, we could define the conditions for obtaining an optimal and durable resolution.

3. Study of the elution gradient allowed us to eliminate artefacts, speed up elution and improve fructose and arabinose separation (fig.2 and table 2). The pH gradient has a strong effect on separation. Its influence is studied in detail (fig. 6).

4. Determining borates (fig. 4) and evaluating $\mathrm{pH}$ at the entrance and exit of the column allowed us to check the true elution gradient and to class carbohydrates into 3 groups according to their resin affinity (fig. 5).

5. Figure 7 shows the limits within which output may vary. When temperature increases from 30 to $65^{\circ} \mathrm{C}$, resolution is better and loss of pressure is decreased, but the volume of elution increases. Beyond $55^{\circ} \mathrm{C}$, carbohydrates are greatly destroyed (fig. 8). This latter temperature is considered as optimal.

6. Carbohydrates are determined at the exit of the column by the sulphuric orcinol method. The automatic determination technique is improved so as to create an optimal sensitivy, to obtain reduced consumption of reactives and high quality results (fig. 3). The coefficient of variation of determination of 20 successive samples of saccharose at $5 \mu \mathrm{g} / \mathrm{ml}$ is $1.2 \mathrm{p}$. Ioo for an average optic density of 0.244 ( $15 \mathrm{~mm}$ flowell).

7. The effect on the colorimetric reaction of constituents of buffer solutions and various products which may interfere is estimated (table 3 ).

8. In such operating conditions, carbohydrates may degrade in the column into various acids and are usually transformed (epimerization). Recovery rates of 12 high-concentration sugars, measured by two chemical methods and a radiochemical method, are constant (table 4). Since reproductibility of the method is satisfactory at various concentrations (tables 5 and 7 ), it may be concluded that if the sugars are partially degraded in the resin, they degrade at a constant rate, no matter what the concentration is. Moreover, they are usually very little transformed (table 4). 
9. No inner standard is added because variation of recovery rates from one chromatography to another are not the same for all sugars. On the contrary, an outer sucrose standard is used to estimate variations resulting from the analytical circuit.

Io. Peak surface was measured by triangulation and electronic integration (table 6). At low carbohydrate concentrations, both methods are inaccurate, the first, however, being preferable. At higher concentrations the two methods are comparable. The amount of sugar cannot be estimated by simple measurement of peak height because the width of the peak in not constant when concentration varies (table 8 ). The width also varies in terms of working time of the column.

I I. Surface-concentration proportionality (fig. 9) is good, except at low concentrations for some carbohydrates. Accuracy of quantitative estimation is satisfactory because the interval of confidence on the surface-concentration proportionality constant is not more than $3.0 \mathrm{p}$. Ioo (table 7). For optimal accuracy it is advisable to analyze solutions having carbohydrate concentrations between those shown in series 2 and 5 of table. 5 .

I2. Reproducibility of retention volume is very good (table 8).

\section{RÉFÉRENCES BIBLIOGRAPHIQUES}

Adachl S., Sugawara H., I963. Separation of ketose and aldose by chromatography on an ionexchange column. Archs. Biochem. Biophys., 100, 468-471.

Angus H. J. F., Bourne F. J., Searle F., Weigel H., I964. Complexes between polyhydroxyl compounds and inorganic oxy-acids. Tungstate complexes of sugars and other cyclic polyhydroxyl compounds. Tetrahedron leters, 55-60.

Arwidi B., Samuelson O., 1964. Sugar separation on ion-exhange resins in mixture with celite. Anal. chim. Acta, 31, 462-466.

Arwid B., Samulason O., r965. Partition chromatography of sugars on ion-exchange resins. Svensk Kem. Tidskr., 77, 84-9o.

Bial M., 1902. Die Diagnose def Pentosurie. Dt. Med. Wschr., 28, 263-264.

BOESEKEN J., I949. The use of boric acid for the determination of the configuration of carbohydrates. Adv. Carbohyd. Chem., 4, I89-210.

Brown A. H., I946. Determination of pentose in the presence of large quantities of glucose. Archs Biochem., 11, 269-278.

BrUCKNER J., I955. Estimation of the monosaccharides by the orcinol-sulfuric acid reaction. Bio. chem. $J, 60,200-205$.

13uhler D. R., Thomas R. C., Wang C. H., 1954. Epimerization and degradation of glucose by ion exchange resin. Analyt. Chem., 26, 1248-1249.

Buhler D. R., Thomas R. C., Christensen B. E., Wang C. H., i955. Epimerization and fragmentation of glucose by quaternary ammonium base type anion-exchange resins. $J . A m$. Chem. Soc., 7y. $48 \mathrm{I}-482$.

Callicoat D. L., Wolszon J. D., i959. Carminic acid procedure for determination of boron. Analyt. Chem., 31, 1434-I 437.

Carubelli R., I966. Transformation of disacharides during borate ion exchange chromatography. Isomerization of lactose into lactulose. Carbohydrate Res., 2, 480-485.

Catravas G. N., r966. New developments in automatic sugar chromatography. Symposium Technicon "Automation in Analytical Chemistry ". I966 (82). Compagnie Technicon, 95, Domont.

Everest D. A., Popiel W. J., I956. Ion exchange studies of solutions of borates. J. Chem. Soc., $3183-3189$.

FLoridi A., 1971. An improved method for the automated analysis of sugars by ion-exchange chromatography. J. Chromatog., 59, 6r-7o.

François C., Marshall R. D., Neuberger A., I962. Carbohydrates in protein. The determination of mannose in hen's egg albumin by radioisotope dilution. Biochem. $J$, 83, 335-34I.

Gottlieb M. H., Gregor H. P., I954. Studies on ion-exchange resins. XI. Activity coefficients of diffusible ions in a strong base anion-exchange resin. J.Am. Chem. Soc, 76, 4639-464r.

Green J. G., 1966. Automated carbohydrate analyzer : experimental prototype. Natn. Cancer Inst. Monogr., 21, 447-467.

Gregor H. P., Belle J., Marcus R. A., I954. Studies on ion-exchange resins. IX. Capacity and specific volumes of quaternary base anion-exchange resins. J. Am. Chem. Soc., 76, I984-I987.

GREGORY D. J., I960. The effect of borate on the carbazole reaction. Archs. Biochem. Biophys., 89, I57-I59.

Hallen A., I960. Chromatographic analysis of sugars as complex borate ions. Acta Chem. Scand., $14,2249-2250$. 
Hough L., Jones J. K. N., Richards E. L., I953. The reaction of amino compounds with sugars. Part II. The action of ammonia on glucose, maltose and lactose. J. Chem. Soc., 2005-2009.

Hough L., Jones J. K. N., Richards E. L., I954. The reaction of amino compounds with sugars. Part III. The action of ammonia on melibiose. J. Chem. Soc. 295-297.

Hough L., Priddle J. E., Theobald R. S., i960. Separation of carbohydrates on anion-exchange resins., $\mathbf{2 8}$, 900 .

Hulme A. C., I953. An action of strongly basic anion-exchange resins and solutions containing sugars. Nature, 171, 610-6II.

Isbell H. S., Brewster J. F., Holt N. B., Frush H. L., I948. Behavior of certain sugars and sugar alcohols in the presence of tetraborates - Correlation of optical rotation and compound formation. J. Res, natn. Bur. Stand., 40, r29-149.

Jolley R. L., Freeman M. L., ig68. Automated carbohydrate analysis of physiologic fluids. Clin. Chem., 14, 538-547.

Jonsson P., SAmuelson O., I966. Automated chromatography of sugars. Sci. Tools, 13, I7-22.

Jonsson P., SAMuelson O., I967. Automated chromatography of sugars on cation-exchange resin. Analyt. Chem., 39, II56-II58.

Kesler R. B., I967. Rapid quantitative anion-exchange chromatography of carbohydrates. Analyt. Chem., 39, I4 $16-1422$.

KнYм J. X., ZILL L. P., I95I. The separation of monosaccharides by ion-exchange. J. Am. Chem. Soc., 173, 2399-2400.

Кнум J. X., Zill $\quad$ L. P., I952. The separation of sugars by ion-exchange. J. Am. Chem. Soc, 74, 20902094.

Lee Y. C., MeKelvy J. F., LANG D., I969. Rapid automatic analysis of sugar components of glycoproteins. II. Neutral sugars. Analyt. Biochem., 24, 567-574.

Lin F. M., Pomeranz Y., i968. Effect of borate on colorimetric determinations of carbohydrates by phenol-sulfuric acid method. Analyt. Biochem., 24, I28-I 3 I.

Lobry de Bruyn C. A., Alberda Van Ekenstein W., I895. In Speck J. C., i958. The Lobry de Bruyn-Alberda Van Ekenstein transformation. Adv. Carbohyd. Chem., 13, 63-10z.

LouRIJSEN-TEYSSEDRE, 1955. Contribution à l'étude des phénomènes de condensation en chimie minérale. IX. Constitution des solutions de borates. Bull. Soc, Chim. Fr., IrII-III7.

Mendel. B., Kemp A., Myers D. K., I954. A colorimetric micromethod for the determination of glucose. Biochem. J., 56, 639-646.

Mendicino J. F., ig60. Effect of borate on the alkali catalysed isomerization of sugars. J. Am. Chem. Soc., 82, 4975-4979.

Montrevil J., Spik G., 1963. Microdosage des glucides. Méthodes colorimétriques de dosage des glurides totaux. Monographie $\mathbf{n}^{\mathbf{0}}$ I, 7. Laboratoire de Chimie Biologique de la Faculté des Sciences de Lille.

Montreuil J., SPik G., Konarska A., I963. Microdosage des glucides. Méthodes chromatographiques de dosages des ases neutres. Monographie $\mathrm{n}^{\circ} 3$, Io-I 4. Laboratoire de Chimie Biologique de la Faculté des Sciences de Lille.

Moore S., Spackman D. H., Stern W. H., I958. Chromatography of amino acids on sulfonated polystyrène resins. Analyt. Chem., 30, Ix85-1 I90.

Mundie C. M., Cheshire M. V., Bacon J. S. D., 1968. An automated procedure for the separation and estimation of sugars in acid hydrolysates of soil. Biochem. J., 108, $5 \mathrm{I}-52$.

Ohms J. I., Zec J., Benson J. R., Patterson J. A., I967. Column chromatography of neutral sugars : operating characteristics and performance of a newly available anion-exchange resin. Analyt. Biochem., 20, 5I-57.

Peterson E. A., Sober H. A., 1959. Variable gradient device for chromatography. Analyt. Chem., 31, $857-862$.

Phillips J. P., Pollard A., 1953. Degradation of sugars on ion-exchange columns of Amberlite IRA $400\left(\mathrm{OH}^{-}\right)$. Nature, 1'ri, $4 \mathrm{I}^{-42}$.

Rebenfeld L., PACsu E., I953. Interconversion and degradation of reducing sugars by anion-exchange resins. J. Am. Chem. Soc., 75, 4370-4372.

Samuelson O., Swendon B., 1962. Partition chromatography on ion-exchange resins. Separation of sugars. Acta. Chem. Scand. 16, 2056-2058.

Samuelson O., Stromberg H., 1968. Partition chromatography of mono-, di- and trisaccharides on ion-exchange resins. Z. Anal. Chem., 236, 506-513.

Saunders R. M., 1968. Separation of sugars on anion-exchange resin. Carbohydrate Res., 7, 76-79.

Schönenberger M., Kellner H., SüdhoF H., Haupt H., r957. Zur Methodik der Hexosebestimmung in Serumproteinen mit Orcin. Hoppe-Seyler's Z. physiol. Chem., 309, 145-157.

Schultze H. E., Schmintberger R., Haupt H., I958. Untersuchungen uber die gebundenen Kohlenhydrate in isolierten Plasmatproteiden. Biochem. Z. 329, 490-507.

Spackman H. D., Stein W. H., Moore S., I958. Automatic recording apparatus for use in the chromatography of amino acids. Analyt. Chem., 30, I I90-г 206.

Speck J. C., 1958. The Lobry de Bruyn-Alberda van Ekenstein transformation. Adv. Carbohyd. Chem., 13, 63-102. 
Syamananda R., Staples R. C., Block R. C., I962. Automatic analysis of sugars separated by column chromatography. Contr. Boyce. Thomson. Inst. Pl. Res., 21, 363-369.

Technicon, 1966. Techniques in amino acid analysis. Monograph $\mathrm{n}^{\circ} \mathrm{I}, \mathrm{147}$. Technicon Instruments Company Ltd. Chertsey, Surrey, England.

Tillmans J., Phillipi K., 1929. Uber den Gehalt der wichtigsten Proteine der Nahrungsmittel an Kolhlenhydrat und über ein kolorimetrisches Verfahren zur quantitativen Bestimmung von stickstoffreiem Zucker in Eiweis. Biochem. Z., 215, 36-60.

Touze A., Auriol P., I97o. Analyse chromatographique de glucides des végétaux, oses et oligosides. Symposium Technicon "Automation in Analytical Chemistry" 1970 (47). Compagnie Technicon. 95. Domont.

Walborg E. F., Christensson L., Gardell S., 1965. An ion-exchange chromatographic method for the separation and quantitative analysis of neutral monosaccharides. Analyt. Biochem., 13, 177I 85 .

WALBorg E. F., LANZ R. S., I968. Separation and quantitation of saccharides by ion-exchange chromatography using boric acid-glycerol buffer. Analyt. Biochem., 22, 123-I33.

WALBorg E. F., RAY D. B., OHRBERG L. E., I969. Ion-exchange chromatography of saccharides : an improved system utilising boric acid-2, 3, butanediol buffers. Analyt. Biochem., 29, 433-440.

WALBorg E. F., Kondo L. E., I97o. Automated system for ion-exchange chromatography of saccharides. Analyt. Biochem., 37, 320-329.

Weber P., Bornstein I., Winzler R. J., ig66. An automated column chromatographic procedure for the fluorometric determination of mixtures of hexoses. Analyt. Biochem., 14, roo-I05.

Wood P. J., Cousins A. R., 1966. The analysis of sucrose, fructose and glucose in tobacco. Symposium Technicon "A utomation in analytical chemistry" 97-101. E. Kawerau, Mediad Inc. New York.

ZERFING R. C., VeENing H., 1966. Anion-exchange chromatography of some carboxylic acids. Analyt. Chem., 38, I312-131\%.

ZILL L. P., Khym J. X., Cheniae G. M., 1953. Further studies on the separation of the borate complexes of sugars and related compounds by ion-exchange chromatography. J. Am. Chem. Soc., 75, I339-I342. 